\title{
The NR1 M3 Domain Mediates Allosteric Coupling in the $N$-Methyl-D-aspartate Receptor
}

\author{
Marie L. Blanke and Antonius M. J. VanDongen \\ Department of Pharmacology and Cancer Biology, Duke University Medical Center, Durham, North Carolina (M.L.B., A.M.J.V.); \\ and Duke-NUS Graduate Medical School, Singapore, Singapore (A.M.J.V.)
}

Received December 4, 2007; accepted April 28, 2008

\begin{abstract}
$N$-Methyl-D-aspartate (NMDA) receptors play a critical role in both development of the central nervous system and adult neuroplasticity. However, although the NMDA receptor presents a valuable therapeutic target, the relationship between its structure and functional properties has yet to be fully elucidated. To further explore the mechanism of receptor activation, we characterized two gain-of-function mutations within the NR1 M3 segment, a transmembrane domain proposed to couple ligand binding and channel opening. Both mutants (A7Q and A7Y) displayed significant glycine-independent currents, indicating that their M3 domains may preferentially adopt a more activated conformation. Substituted cysteine modification experiments revealed that the glycine binding clefts of both $A 7 Q$ and $A 7 Y$ are inaccessible to modifying reagents and resistant to competitive antagonism. These data suggest that
\end{abstract}

perturbation of M3 can stabilize the ligand binding domain in a closed cleft conformation, even in the absence of agonist. Both mutants also displayed significant glutamate-independent current and insensitivity to glutamate-site antagonism, indicating partial activation by either glycine or glutamate alone. Furthermore, $A 7 Q$ and $A 7 Y$ increased accessibility of the NR2 M3 domain, providing evidence for intersubunit coupling at the transmembrane level and suggesting that these NR1 mutations dominate the properties of the intact heteromeric receptor. The equivalent mutations in NR2 did not exhibit comparable phenotypes, indicating that the NR1 and NR2 M3 domains may play different functional roles. In summary, our data demonstrate that the NR1 M3 segment is functionally coupled to key structural domains in both the NR1 and NR2 subunits.
$N$-methyl-D-aspartate (NMDA) receptors are a critical component of the glutamatergic signaling system, responsible for generating excitatory synaptic currents that control neural transmission (Collingridge and Bliss, 1995). Long-term changes in synaptic strength, believed to underlie learning and memory formation, are induced by NMDA receptor-mediated calcium influx (Malenka and Nicoll, 1999). Consequently, receptor hypofunction has been implicated in many neurological disorders, ranging from cognition defects to schizophrenia and Alzheimer's disease (Weeber and Sweatt, 2002; Emamian et al., 2004; Hynd et al., 2004). Overstimulation of NMDA receptors, which can result from excessive glutamate release after stroke or traumatic brain injury, triggers excitotoxic cell death (Mody and MacDonald, 1995), thus illustrating the importance of precise regulation.

This work was supported by National Institute of Health grants F31NS053030-01 (to M.L.B.) and R01-MH61506 (to A.M.J.V.D.)

Article, publication date, and citation information can be found at http://molpharm.aspetjournals.org.

doi:10.1124/mol.107.044115.
NMDA receptor activation requires concurrent glutamate release and membrane depolarization, as well as binding of a coagonist, glycine (Mayer et al., 1984; Kleckner and Dingledine, 1988). In addition, NMDA receptors are regulated by a variety of endogenous modulators, including protons, $\mathrm{Zn}^{2+}$, polyamines, and neurosteroids (Dingledine et al., 1999; Stoll et al., 2007). At the clinical level, pharmacological inhibition of glutamate receptors has been used for anesthesia and treatment of stroke and Alzheimer's disease (Muir, 2006). Given the broad therapeutic potential of NMDA receptor antagonists and their diverse pharmacology, more detailed structure-function studies should prove extremely valuable.

NMDA receptors generally form as heteromultimers of NR1 and NR2 subunits, which bind glycine and glutamate, respectively (Schorge and Colquhoun, 2003). Each subunit has the same modular domain structure, including a ligand binding domain (LBD), three putative transmembrane segments (M1, M3, and M4), and a pore-forming M2 loop (Fig. 1) (Mayer, 2006). Recent crystal structures of the LBDs have helped elucidate ligand binding and cleft closure (Furukawa

ABBREVIATIONS: NMDA, N-methyl-D-aspartate; LBD, ligand binding domain; GluR, glutamate receptor; LC-MS, liquid chromatography-mass spectrometry; Lobar, low-barium Ringer's solution; MTSEA, methanethiosulfonate; DCK, 5,7-dichlorokynurenic acid; PS, pregnenolone sulfate; DCS, D-cycloserine; HA-966, 3-amino-1-hydroxy-2-pyrrolidinone; WT, wild type; DTNB, dithionitrobenzoic acid; APV, 2-amino-5-phosphonovalerate; MK-801, $5 \mathrm{H}$-dibenzo[a,d]cyclohepten-5,10-imine (dizocilpine maleate); $P_{\mathrm{o}}$, open probability. 
et al., 2005), whereas single-channel studies have provided a detailed analysis of gating behavior (Banke and Traynelis, 2003; Auerbach and Zhou, 2005); however, structural information on the transmembrane region remains sparse, and the mechanism linking binding of agonists, antagonists, and modulators to channel gating is not fully understood.

Previous studies have suggested that the M3 segment functions as a transduction element, coupling ligand binding to channel opening (Jones et al., 2002; Yuan et al., 2005). M3 contains the highly conserved SYTANLAAF motif, and many cysteine mutations in this region exhibit state-dependent accessibility, implying that M3 movement occurs in response to activation. Recent studies have also shown that M3 is the only transmembrane domain contributing to the deepest portion of the pore, supporting a prominent role in gating (Sobolevsky et al., 2007). In vivo, the lurcher mouse phenotype, a neurodegenerative condition caused by excitotoxic cell death, has been attributed to a constitutive mutation within the GluR 22 SYTANLAAF region (A8T) (Zuo et al., 1997), and introduction of the lurcher mutation into other glutamate receptors results in numerous gain-of-function phenotypes, including increased agonist potency, slower deactivation rates, and inhibition of desensitization (Kohda et al., 2000; Klein and Howe, 2004; Hu and Zheng, 2005; Schmid et al., 2007).

To further evaluate how M3 couples to surrounding structural domains, we assessed the effect of two novel gain-offunction mutants on channel activation. Based on substituted cysteine accessibility, we conclude that shifting the activation equilibrium of M3 stabilizes the glycine binding domain in a closed cleft conformation. Interestingly, the equivalent mutations in NR2 failed to produce gain-offunction phenotypes, suggesting different functional roles for the two subunits. Finally, we demonstrate intersubunit coupling of these effects to the glutamate binding domain, NR2 M3, and channel pore, indicative of an allosteric coupling mechanism.

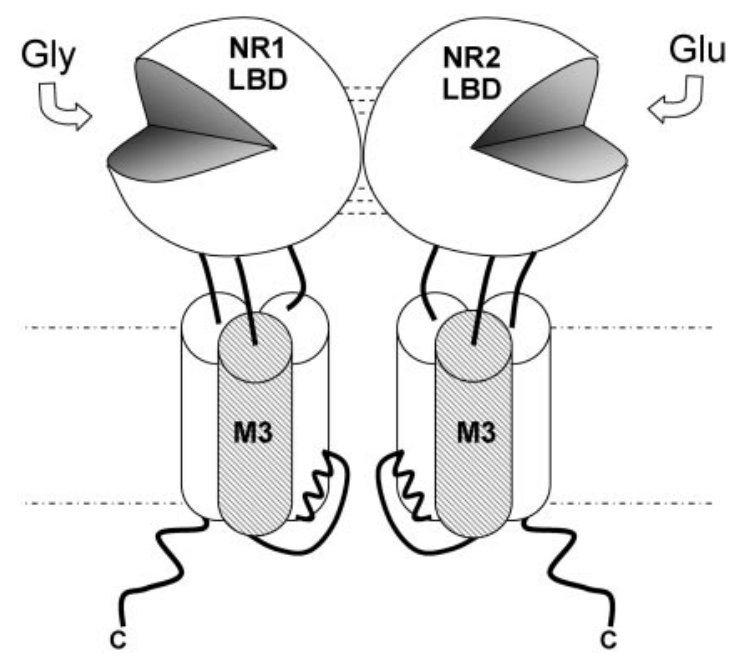

Fig. 1. Topology of the M3 domain. Diagram illustrating modular domain structure of the NMDA receptor. The NR1 and NR2 LBDs bind glycine and glutamate, respectively. Dashed lines represent the intersubunit LBD interface. Transmembrane segments M1, M3, and M4 are illustrated as cylinders, with the M2 loop shown lining the pore. For clarity, only two of four subunits are depicted, and the amino-terminal domains have been omitted.

\section{Materials and Methods}

Site-Directed Mutagenesis. Mutations in NR1 were generated using the megaprimer polymerase chain reaction method, as described previously (Wood et al., 1995), and subsequently confirmed by DNA sequencing of the cloned region. cRNA was prepared from linearized plasmids by in vitro transcription (Wood et al., 1995). Amino acids in both NR1 and NR2 are numbered from the initiator methionine. The NR2 subunit used in all experiments is an NR2A construct with a modified 5 '-untranslated region, previously shown to increase expression (Wood et al., 1996).

Oocyte Preparation and Injection. Stage V-VI oocytes were surgically removed from Xenopus laevis frogs under tricaine anesthesia, followed by manual defolliculation and treatment with collagenase type I (Invitrogen, Gaithersburg, MD) (Jones et al., 2002). All frog work was carried out in accordance with the National Institutes of Health Guide for the Care and Use of Laboratory Animals. Oocytes were injected with a 1:1 ratio of NR1 and NR2 cRNA (75 nl of a 10-100 ng/ $\mu \mathrm{l}$ solution), transferred to SOS buffer, and incubated at $19^{\circ} \mathrm{C}$. SOS buffer consisted of $100 \mathrm{mM} \mathrm{NaCl}, 2 \mathrm{mM} \mathrm{KCl}, 1.8 \mathrm{mM}$ $\mathrm{CaCl}_{2}, 1 \mathrm{mM} \mathrm{MgCl}_{2}$, and $5 \mathrm{mM}$ HEPES, pH-adjusted to 7.6 with $\mathrm{NaOH}$ and supplemented with $100 \mu \mathrm{g} / \mathrm{ml}$ gentamicin.

Two-Electrode Voltage-Clamp Electrophysiology. Functional expression was assessed 2 to 6 days after injection using a two-electrode voltage-clamp amplifier (OC-725; Warners Instrument, Hamden, CT). The extracellular recording solution consisted of low-barium Ringer's solution (Lobar: $100 \mathrm{mM} \mathrm{NaCl}, 5 \mathrm{mM} \mathrm{KCl}, 0.5 \mathrm{mM} \mathrm{BaCl}_{2}, 10 \mathrm{mM}$ HEPES, and $10 \mu \mathrm{M}$ EDTA), pH-adjusted to 7.35 with Tris base and maintained at room temperature. Barium was used as the divalent cation to minimize secondary activation of calcium-activated $\mathrm{Cl}^{-}$currents (Leonard and Kelso, 1990). EDTA was included to chelate trace amounts of the soft metal divalent cations $\mathrm{Cd}^{2+}$ and $\mathrm{Zn}^{2+}$, which have been reported to contaminate buffer solutions (Paoletti et al., 1997) and inhibit the NMDA receptor by binding to a high-affinity site (Low et al., 2000; Paoletti et al., 2000). EDTA also removes a zinc-dependent component of desensitization (Zheng et al., 2001). Maximal current response was elicited by application of $100 \mu \mathrm{M}$ glycine and $100 \mu \mathrm{M}$ L-glutamate, unless stated otherwise. Voltage-clamp recordings were performed in a perfusion chamber (Warner Instruments) optimized for laminar flow, and solution changes were accomplished using a gravityfed, computer-controlled perfusion system, at a flow rate of $\sim 15 \mathrm{ml} / \mathrm{min}$ (Jones et al., 2002). Oocytes were impaled with low-resistance glass microelectrodes (0.5-2.0 M $\Omega$ ) filled with $3 \mathrm{M} \mathrm{KCl}$ and maintained at a holding potential of $-60 \mathrm{mV}$. Data acquisition and voltage control were performed with pClamp hardware and software (Molecular Devices, Sunnyvale, CA). A7C and A714C thiol modification experiments were performed as described in Jones et al. (2002) and Wood et al. (1997), respectively.

Measurement of Contaminating Glycine Concentration. The concentration of glycine in our Lobar solution, both in the presence and absence of glutamate, was evaluated via liquid chromatography-mass spectrometry (LC-MS) using an ABI QStar electrospray mass spectrometer (Applied Biosystems). The samples were run in positive mode, using a reverse phase column and 10 to $95 \%$ acetonitrile, $0.1 \%$ trifluoroacetic acid gradient. Glycine peaks $(\mathrm{m} / z 76)$ were not observed in the total ion, total wavelength, or extracted ion chromatograms at a detection threshold of $1 \mathrm{pM}$.

Curve Fitting and Statistics. Concentration-response curves were obtained by fitting data with a modified Hill equation: $R / R_{\max }=\mathrm{Y}_{0}+$ $\left(1-Y_{0}\right) /\left[1+\left(\mathrm{EC}_{50} / A\right)^{n \mathrm{H}}\right.$, where $R$ is the response for the given agonist concentration $(A), R_{\max }$ is the maximum response, $n_{\mathrm{H}}$ is the Hill coefficient, $\mathrm{EC}_{50}$ is the concentration midpoint, and $Y_{0}$ is the $\mathrm{y}$-intercept. Parameters were optimized by minimizing the residual sum of squares using the Solver function in Excel (Microsoft, Redmond, WA). Antagonist $K_{\mathrm{D}}$ values were estimated using the Cheng-Prusoff equation: $K_{\mathrm{D}}=$ $\mathrm{IC}_{50} /\left[1+\left(A / \mathrm{EC}_{50}\right)\right]$, where $K_{\mathrm{D}}$ is the dissociation constant and $A$ is agonist concentration. Each concentration point represents four to eight oocytes, and error bars indicate S.E.M. All other data are presented as 

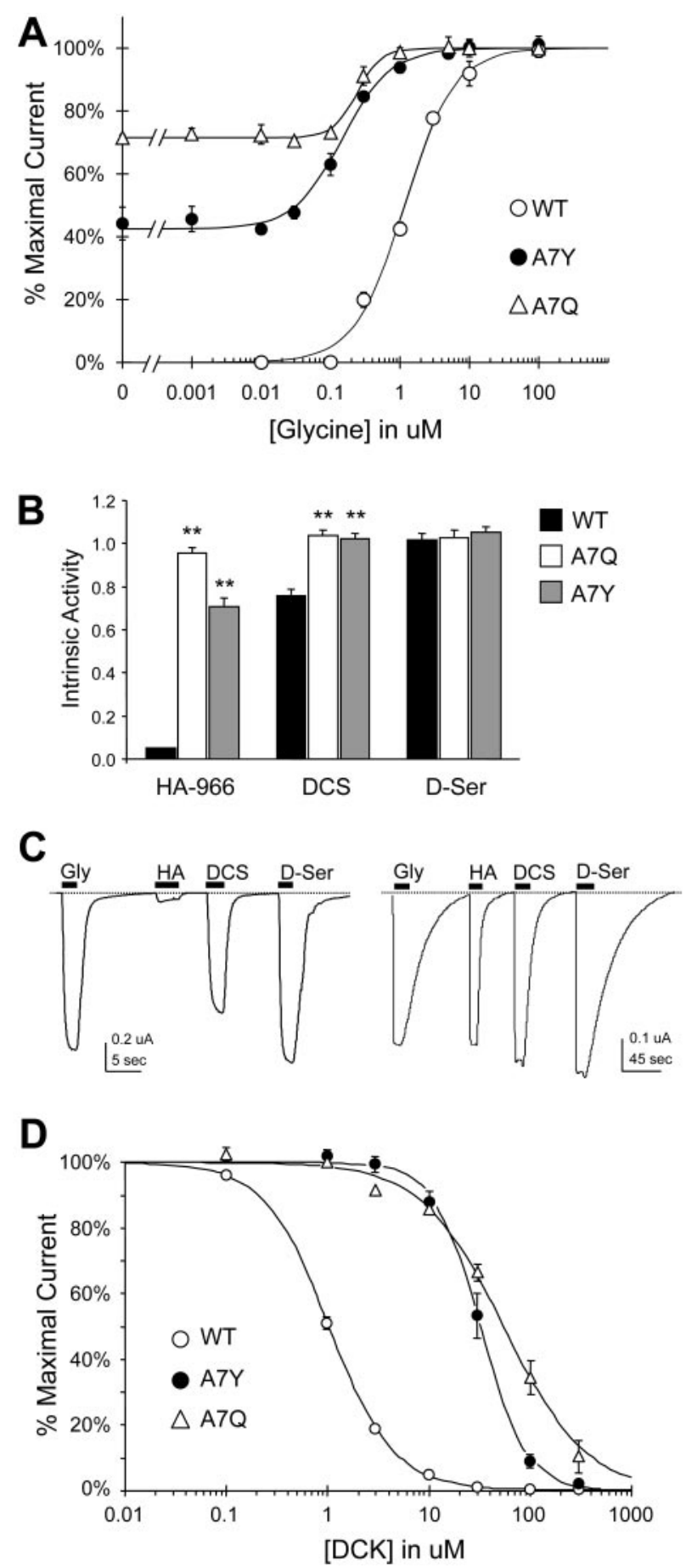

Fig. 2. Altered activation equilibrium in NR1 A7Q and A7Y. A, glycine concentration-response curves in the presence of $100 \mu \mathrm{M}$ L-glutamate. Both A7Q $(\triangle)$ and A7Y $(\bullet)$ exhibited significant glycine-independent current (71 and $44 \%$, respectively) and increased sensitivity to glycine. Concentration midpoints were $0.24 \mu \mathrm{M}$ for $\mathrm{A} 7 \mathrm{Q}$ and $0.15 \mu \mathrm{M}$ for $\mathrm{A} 7 \mathrm{Y}$, compared with $1.16 \mu \mathrm{M}$ for WT. Hill coefficients were 2.5 for A7Q and 1.2 for A7Y, compared with 1.2 for WT. B, response to glycine-site partial agonists, calculated as a fraction of full agonist response. HA-966 (500 $\mu \mathrm{M}), 1 \mathrm{mM}$ DCS, and $100 \mu \mathrm{M}$ D-serine were coapplied with $100 \mu \mathrm{M}$ glutamate. Both A7Q and A7Y displayed increased activity in response to HA-966 and DCS, but not D-serine. Intrinsic activities for WT, A7Q, and A7Y were as follows: HA-966: $0.05 \pm 0.00,0.96 \pm 0.02$, and $0.71 \pm 0.04$; DCS: $0.76 \pm 0.03,1.04 \pm 0.02$, and $1.02 \pm 0.02$; and D-Ser: $1.02 \pm 0.03$, $1.03 \pm 0.07$, and $1.06 \pm 0.03$. C, representative whole cell traces illustrating the response of WT (left) and A7Q (right) to glycine-site partial agonists in the presence of $100 \mu \mathrm{M}$ glutamate. A7Q receptors exhibit increased partial agonist responses and markedly slow deactivation. D, mean \pm S.E.M. from five to 12 oocytes and are analyzed statistically using one-way analysis of variance. For multiple comparisons, the data were initially subjected to a global analysis of variance incorporating all factors and measurements, and if this test showed a strong interaction between mutant and agonist response $(p<0.001)$, data were subdivided by agonist for lower order tests. Fisher's protected least significant difference test was then applied to compare the effects of the mutation on each response. Statistical significance is indicated with an asterisk for $p<0.05$ (significant) or a double asterisk for $p<0.01$ (highly significant).

Molecular Modeling. NR1 A714C structures were generated using Swiss PDB Viewer (http://expasy.org/spdbv), and the resulting molecular graphics were rendered in POV-Ray version 3.6 (http:// www.povray.org).

Reagents. All chemicals were purchased from Sigma-Aldrich (St. Louis, MO), with the exception of methanethiosulfonate (MTSEA) (Toronto Research Chemicals, North York, ON, Canada), glycine (EMD Biosciences, San Diego, CA), and L-glutamate (Gibco BRL, Carlsbad, CA). 5,7-Dichlorokynurenic acid (DCK) and pregnenolone sulfate (PS) stock solutions were initially made in dimethyl sulfoxide and diluted to their final concentrations in Lobar. For those experiments, dimethyl sulfoxide was added to all recording solutions to maintain a standard concentration.

\section{Results}

We previously reported that cysteine substitution at position A7 of the SYTANLAAF motif, a strictly conserved region located in the extracellular portion of $\mathrm{M} 3$, results in a unique activation phenotype (Jones et al., 2002). Introduction of the A7C mutation into either the NR1 or NR2A subunit produces a receptor with state-dependent accessibility, assessed by treatment with the thiol-specific compound MTSEA. MTSEA modification of the introduced cysteine, which occurs only in the presence of agonist, stabilizes the receptor in an activated state. These results suggest that M3 is directly involved in coupling ligand binding to channel opening. In the present study, we have further investigated the role of M3 in NMDA receptor activation by making additional substitutions at the A7 position.

A7Q and A7Y Shift M3 to an Activated Conformation. Initially, a set of representative amino acids was introduced at position A7 of the NR1 subunit and evaluated according to glycine sensitivity. Substitution of alanine with two residues, glutamine and tyrosine, resulted in a particularly striking phenotype; these mutants both displayed increased glycine sensitivity and significant activation in the absence of glycine (Fig. 2A). A7Q and A7Y were 71 and $44 \%$ activated, respectively, by glutamate alone, and displayed 5 - to 8 -fold increases in glycine potency. To determine whether the increased sensitivity was specific to glycine, we also tested responses to several other glycine-site agonists: HA-966, D-cycloserine (DCS), and $\mathrm{D}$-serine. The response of both mutants to D-serine, a full agonist, was similar to that of wild type (Fig. 2, B and C). However, DCS, a partial agonist with an intrinsic activity of 0.76 in wild-type receptors, became a full agonist in both A7 mutants. And HA-966, a very weak partial agonist with an intrinsic

inhibition by DCK, a competitive glycine-site antagonist, in the presence of $10 \mu \mathrm{M}$ glycine and $100 \mu \mathrm{M}$ glutamate. Both mutants were significantly less sensitive to DCK inhibition. $\mathrm{IC}_{50}$ values were $51.5 \mu \mathrm{M}$ for $\mathrm{A} 7 \mathrm{Q}$ and $31.7 \mu \mathrm{M}$ for A7Y, compared with $1.04 \mu \mathrm{M}$ for WT. Hill coefficients were 1.1 for A7Q, 2.3 for A7Y, and 1.5 for WT. Calculated $K_{\mathrm{D}}$ values were as follows: A7Q (1.21), A7Y (0.47), and WT (0.11). *, $p<0.05$ (significant); **, $p<0.01$ (highly significant). 
activity of only 0.05 , elicited responses of 96 and $71 \%$ from A7Q and A7Y, respectively. Because both mutants exhibited increased sensitivity to glycine-site agonists, we also tested the effectiveness of DCK, a competitive glycine-site antagonist. Both A7Q and A7Y were significantly less sensitive to DCK inhibition, shifting the concentration-response curve $\mathrm{IC}_{50}$ value from 1.0 to 51.5 and $31.7 \mu \mathrm{M}$, respectively (Fig. 2D). Dissociation constants calculated from these data revealed an 11-fold increase for A7Q and a 4.3-fold increase for A7Y. Thus, these mutant receptors seem to be poised in a more readily activated state than their wild-type counterparts, possibly reflecting a shifted activation equilibrium. Because both mutants can still be blocked by high DCK concentrations, our results suggest that channel activation remains dependent on LBD closure. Position A7 is located within the M3 transmembrane domain, and not in proximity to the glycine binding domain, so it is unlikely that this residue participates directly in agonist binding or LBD closure. Instead, we interpret the altered activation equilibrium as a shift in position or conformation of the M3 domain, consistent with its proposed role as a transduction element, with subsequent allosteric effects on the LBD.

Many studies have reported trace glycine contamination of buffer solutions, ranging from 10 to $50 \mathrm{nM}$ (Johnson and Ascher, 1987; Lerma et al., 1990; Zhang et al., 1997; Paudice et al., 1998). In our glycine concentration-response experiments, WT receptors exhibited no response to $10 \mathrm{nM}$ glycine in the presence of $100 \mu \mathrm{M}$ glutamate $(0.00 \pm 0.00$; Fig. $2 \mathrm{~A})$, and combining our A7Q mutant with NR1 T518C, previously shown to decrease glycine affinity by more than 100 -fold (Kalbaugh et al., 2004), resulted in an identical glutamateonly response ( $71 \pm 4 \%$; data not shown). However, to ensure that the agonist-independent responses observed in our mutants were not the result of contaminating glycine, our buffer and glutamate-only solutions were assayed via LC-MS, which determined glycine levels to be below a detection level of $1 \mathrm{pM}$. Given that the concentration midpoints of $\mathrm{A} 7 \mathrm{Q}$ and A7Y were 15 and $24 \mathrm{nM}$, respectively, the gain-of-function phenotypes of these mutants are unlikely to be caused by contaminating glycine. The possibility remains that glycine leakage from within the oocytes could form a local concentration gradient around the recording electrode; however, the A7Q/T518C phenotype argues against this hypothesis.

Stabilization of the LBD in a Closed Cleft State. To further examine the behavior of the A7Q and A7Y glycine binding domains, we constructed double mutants with NR1 A714C, a previously characterized reporter of LBD closure located within the glycine binding cleft (Wood et al., 1997). The cysteine residue is accessible in the open cleft conformation but protected from modification after cleft closure (Fig. 3B). Application of $500 \mu \mathrm{M}$ dithionitrobenzoic acid (DTNB), a thiol-modifying reagent, rapidly and irreversibly inhibits A714C-containing receptors in the absence of glycine (Fig. 3A). Addition of glycine to the DTNB solution dramatically slows the time course of inhibition (50\% inhibition after 5 min), reflecting decreased accessibility of A714C upon closure of the glycine binding cleft. The competitive antagonist DCK does not protect A714C from modification, and partial agonists offer some protection but are less effective than glycine; therefore, the degree of accessibility is thought to reflect the extent of LBD closure (Wood et al., 1997). To assess the LBD conformation of our A7 mutants, A7Q and $\mathrm{A} 7 \mathrm{Y}$ were combined with the $\mathrm{A} 714 \mathrm{C}$ reporter and evaluated for cysteine accessibility. Both A714C/A7Q and A714C/A7Y were completely resistant to DTNB modification in the absence of glycine, indicating that the glycine binding clefts of these mutants adopt an inaccessible conformation. Because glycine can still activate these receptors beyond their glutamate-only response, and DCK remains inhibitory at low glycine concentrations (Fig. 2C), their LBDs are not locked in a fully activated position; more likely, these domains are preferentially stabilized in a closed cleft conformation. Remarkably, the complete lack of accessibility suggests that the LBDs of both mutants, in the absence of agonist, adopt a more stable closed cleft state than a fully liganded WT LBD. This result provides a structural basis for the left-shifted concentration-response curves observed in both mutants, because LBD stability is an important determinant of apparent affinity (Robert et al., 2005). Furthermore, stabilization of

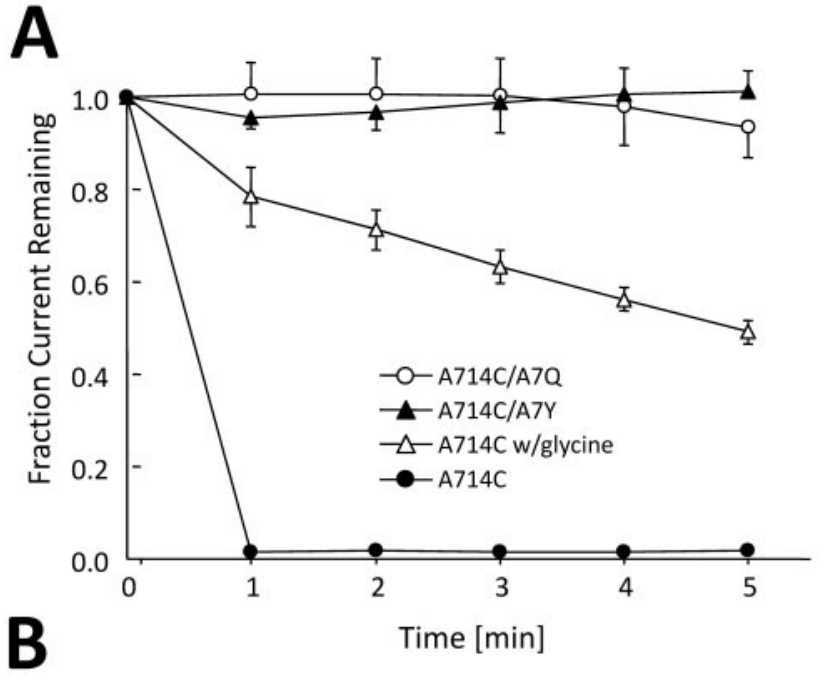

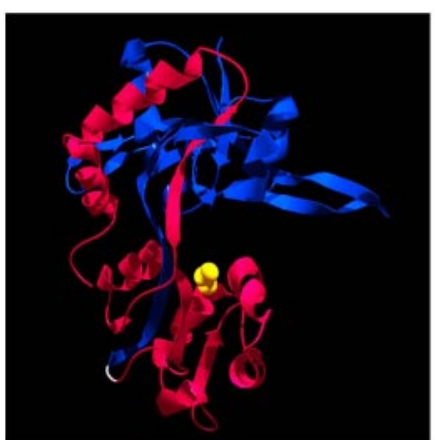

A714C

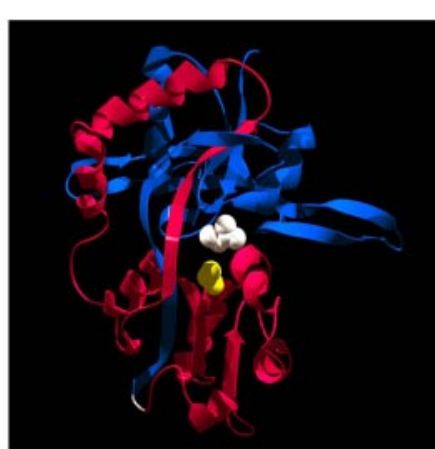

A714C + Glycine
Fig. 3. Decreased accessibility of the glycine binding cleft in $A 7 Q$ and A7Y-containing receptors. A, time course of inhibition by DTNB is shown for receptors containing $\mathrm{A} 714 \mathrm{C}$, a reporter of ligand binding domain closure. Points represent full agonist-induced current, measured at 1-min intervals. Treatment with $500 \mu \mathrm{M}$ DTNB for 1 min resulted in rapid and irreversible inhibition ( ), which was substantially slowed by the presence of $100 \mu \mathrm{M}$ glycine $(\triangle)$. A714C receptors containing A7Q or A7Y, however, were insensitive to DTNB inhibition even in the absence of glycine ( $\bigcirc$ and $\boldsymbol{\Delta}$, respectively), indicating that the ligand binding domains of these receptors are preferentially stabilized in a closed cleft conformation. Percentage of inhibition after $5 \mathrm{~min}$ : A714C, $98 \pm 0.1 \%$; A714C with glycine, $49 \pm 2 \%$; A7Q/A714C, $6.7 \pm 6.7 \%$; and A7Y/A714C, $-1 \pm 4 \%$. B, structures of the NR1 A714C ligand binding domain in the open and closed cleft conformations. A714C is highlighted in yellow, and the bound glycine is depicted in white. The S1 lobe is shown in blue, S2 is shown in pink, and the GT linker is shown in gray. The open and closed conformations are based upon accession codes 1PBQ and 1PB7, respectively. 

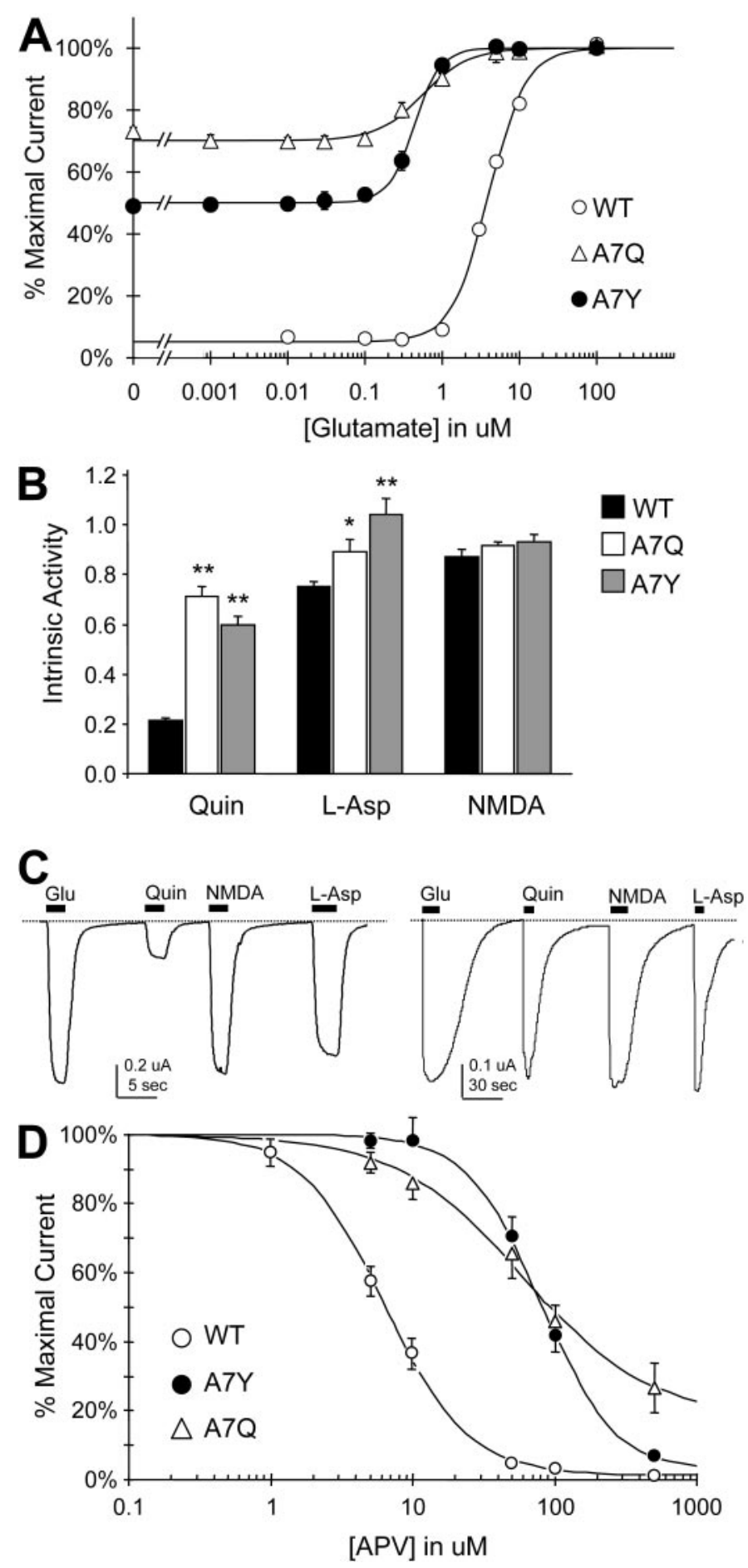

Fig. 4. A7Q and A7Y affect the NR2 ligand binding domain. A, glutamate concentration-response curves in the presence of $100 \mu \mathrm{M}$ glycine. Both A7Q $(\triangle)$ and A7Y $(\bullet)$ exhibited significant glutamate-independent current (73 and $49 \%$, respectively) and increased sensitivity to glutamate. Concentration midpoints were $0.57 \mu \mathrm{M}$ for A7Q and $0.45 \mu \mathrm{M}$ for A7Y, compared with $4.05 \mu \mathrm{M}$ for WT. Hill coefficients were 1.5 for A7Q, 2.6 for A7Y, and 1.9 for WT. B, response to glutamate-site partial agonists, calculated as a fraction of full agonist response. Quinolinic acid (10 mM; Quin), 1 mM NMDA, and $100 \mu \mathrm{M}$ L-aspartate (L-Asp) were coapplied with $100 \mu \mathrm{M}$ glycine. Both mutants displayed increased activity in response to quinolinic acid and L-aspartate, but not NMDA. Intrinsic activities for WT, A7Q, and A7Y were as follows: Quin: $0.22 \pm 0.01,0.71 \pm 0.04$, and $0.60 \pm 0.03$; L-Asp: $0.75 \pm 0.02,0.89 \pm 0.05$, and $1.04 \pm 0.06$; and NMDA: $0.87 \pm 0.03,0.91 \pm$ 0.02 , and $0.93 \pm 0.03$. C, representative whole cell traces illustrating the response of WT (left) and A7Q (right) to glutamate-site partial agonists in the closed cleft state is consistent with the glycine-independent current and reduced DCK sensitivity seen in both mutants and provides strong evidence for reciprocal coupling between M3 and the LBD. Allosteric cooperativity is expected to be reciprocal, so these results are not necessarily surprising (Li and Lester, 1997); however, although numerous studies have documented the positive allosteric effect of LBD conformational changes on the M3 domain, the reverse interaction had not yet been conclusively demonstrated for glutamate receptors, due to the difficulty of assessing structural changes in the LBD.

Coupling of the NR1 M3 to the NR2 Ligand Binding Domain. Because NMDA receptors form as heteromeric complexes, we next examined whether the altered phenotype of A7Q and A7Y affects the NR2 subunit. Concentration-response curves revealed that both mutants have an increased sensitivity for glutamate, relative to wild-type receptors (Fig. 4A). Even more striking, however, was the significant glutamate-independent current exhibited by both A7Q and A7Y (73 and 49\%, respectively). Thus, these receptors can be partially activated by either glycine or glutamate, even though the mutations are only present in the M3 domain of the glycine binding subunit. We also examined the response of both mutants to several other glutamate-site agonists: quinolinic acid, NMDA, and L-aspartate (Fig. 4, B and C). A7Q and A7Y responses to NMDA were similar to WT, but L-aspartate and quinolinic acid elicited significantly increased responses from both mutants. It is noteworthy that the WT intrinsic activity of quinolinic acid (0.22) increased to 0.72 in $\mathrm{A} 7 \mathrm{Q}$ and 0.60 in $\mathrm{A} 7 \mathrm{Y}$. In addition, both mutants were characterized by their susceptibility to the competitive glutamate-site antagonist 2-amino-5-phosphonovalerate (APV), which displayed a reduced effectiveness similar to DCK (Fig. 4D). APV IC I0 $_{50}$ values increased to $86.4 \mu \mathrm{M}$ for A7Q and $83.5 \mu \mathrm{M}$ for $\mathrm{A} 7 \mathrm{Y}$, compared with the wild-type value of 6.2 $\mu \mathrm{M}$. Dissociation constants calculated for $\mathrm{A} 7 \mathrm{Q}$ and $\mathrm{A} 7 \mathrm{Y}$ increased by 2.6- and 2.0-fold, respectively. Taken together, these data suggest that altering the conformational state of the NR1 M3 affects not only the NR1 LBD, but the NR2 LBD as well, indicating a remarkable degree of positive cooperativity between LBDs that have been reported to exhibit negative cooperativity (Regalado et al., 2001). Although A7Q and A7Y seem to favor the closed cleft state, both mutants can be completely inhibited by high concentrations of DCK, indicating that closure of the glycine binding domains is still necessary for channel activation. Most likely, the same can be said for the glutamate binding domain, because APV fully inhibits both mutants; however, the degree of cleft closure in the presence of APV has not yet been determined for wild-type NR2.

One possibility is that the glutamate binding domain is stabilized in a closed cleft conformation in the absence of agonist, as was observed for the glycine binding domain. This hypothesis is supported by the glutamate-independent current, decreased sensitivity to APV, and the striking similarity of these results to those obtained for the glycine binding domain. Unfortunately, a reporter of glutamate binding domain accessibility has not yet

the presence of $100 \mu \mathrm{M}$ glycine. D, inhibition by APV, a competitive glutamate-site antagonist, in the presence of $100 \mu \mathrm{M}$ glycine and $10 \mu \mathrm{M}$ glutamate. Both mutants were significantly less sensitive to APV inhibition. $\mathrm{IC}_{50}$ values were $86.4 \mu \mathrm{M}$ for $\mathrm{A} 7 \mathrm{Q}$ and $83.5 \mu \mathrm{M}$ for $\mathrm{A} 7 \mathrm{Y}$, compared with 6.22 $\mu \mathrm{M}$ for WT. Hill coefficients were 0.8 for A7Q, 2.1 for A7Y, and 1.3 for WT. Calculated $K_{\mathrm{D}}$ values were as follows: A7Q (4.66), A7Y (3.60), and WT (1.79). *, $p<0.05$ (significant); **, $p<0.01$ (highly significant). 
been developed, so this possibility cannot be tested directly. Another possible explanation is that glycine could behave as a partial agonist at the NR2 subunit, as has been suggested by a previous study (Jones et al., 2002). However, APV can still block both A7Q and A7Y in the presence of $100 \mu \mathrm{M}$ glycine (Fig. 4D), so glycine binding to the NR2 LBD is unlikely to fully account for this phenotype. Glutamate has no affinity for the glycine binding domain, due to steric clashes within the cleft (Furukawa and Gouaux, 2003), so the reverse scenario would not be plausible.

Cooperativity between the NR1 and NR2 M3 Domains. Because A7Q and A7Y produced intersubunit effects on the NR2 LBD, we next examined the conformational state of the NR2 M3 domain. Both mutants were coexpressed with NR2A7C, a well characterized reporter of M3 accessibility (Jones et al., 2002). MTSEA modification of A7C is activation-dependent and thus occurs only in the presence of both glycine and glutamate. Several studies have suggested that A7C modification shifts M3 to its maximally activated position, increasing channel open probability $\left(P_{\mathrm{o}}\right)$ to approximately 1.0 . Therefore, the degree of potentiation is believed to correlate inversely with channel $P_{\mathrm{o}}$ (Chen et al., 2005; Yuan et al., 2005). Consistent with previous reports, NMDA receptors containing wild-type
NR1 and NR2-A7C were potentiated significantly by MTSEA modification (Fig. 5A). In contrast, when $\mathrm{A} 7 \mathrm{Q}$ and $\mathrm{A} 7 \mathrm{Y}$ were coexpressed with NR2-A7C, application of MTSEA with glycine and glutamate yielded no current potentiation (Fig. 5, B and C). The observed reversible inhibition most likely represents open channel block, which is also seen in wild-type receptors with no substituted cysteines (Jones et al., 2002). In addition, both A7Q and A7Y exhibited a small degree of irreversible inhibition (7 and $11 \%$, respectively), indicating that covalent modification has a slight inhibitory effect on these mutants. The lack of potentiation could indicate that NR2-A7C is inaccessible to modification, or that NR2-A7C is fully accessible but unaffected by modification, either because the mutant receptors are fully activated or because modification has no effect on these mutants. To distinguish between these possibilities, we repeated the same protocol with each agonist alone, which should partially activate our mutants. Both A7Q and A7Y displayed significant MTSEA potentiation after either glycine or glutamateonly activation (Fig. 5, E and F), indicating that NR2-A7C is still accessible to modification and that modification still potentiates these receptors. To confirm the correlation between accessibility and channel activation, we compared the percentage of glycine- and glutamate-only current with the resulting de-
A

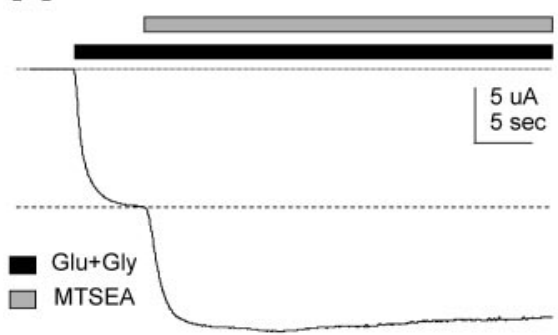

$\mathrm{NR} 1+\mathrm{NR} 2 \mathrm{~A}-\mathrm{A} 7 \mathrm{C}$

D

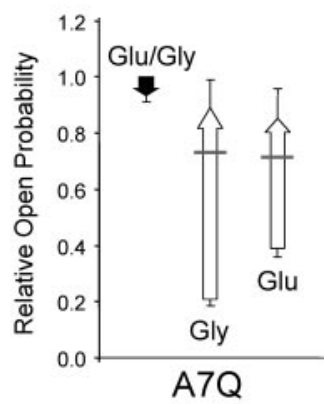

B

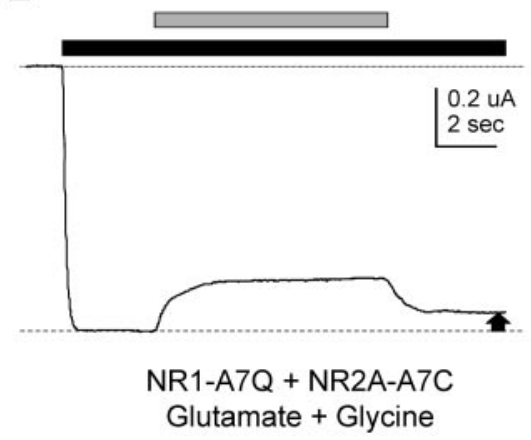

E

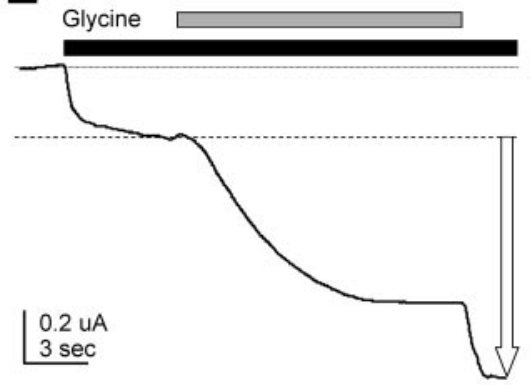

NR1-A7Q + NR2A-A7C

No Glutamate

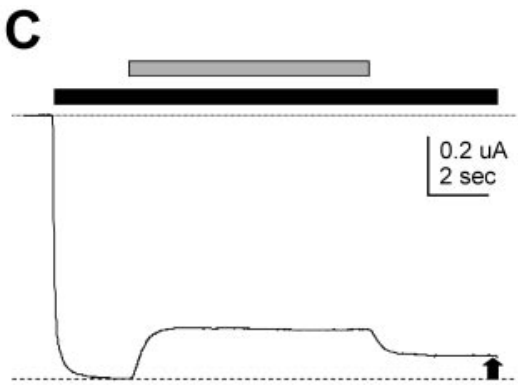

NR1-A7Y + NR2A-A7C

Glutamate + Glycine

F

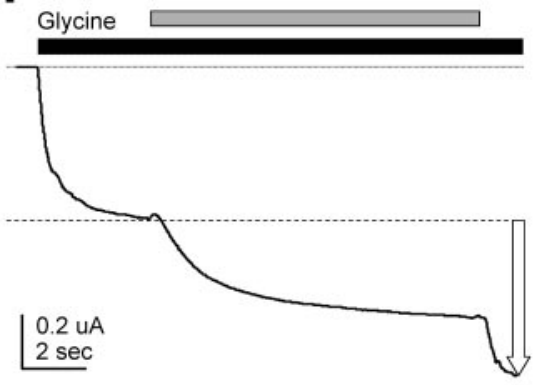

NR1-A7Y + NR2A-A7C No Glutamate

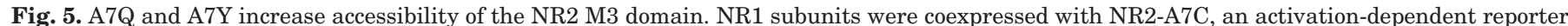

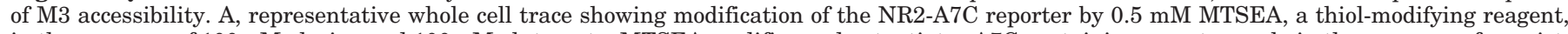

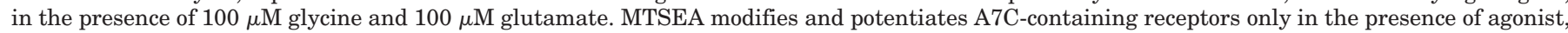

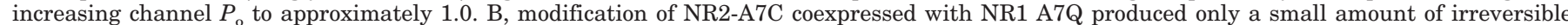

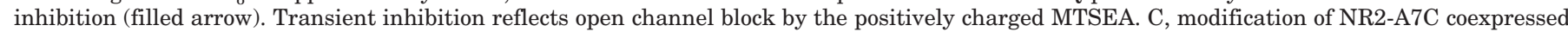

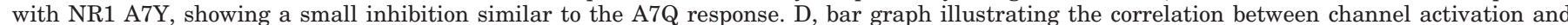

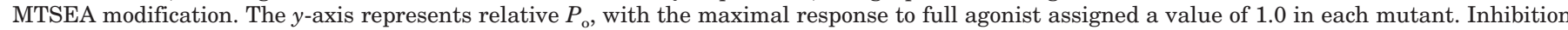

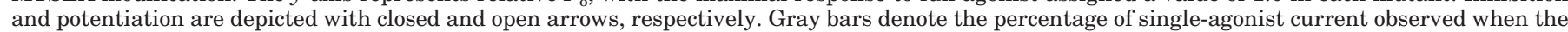

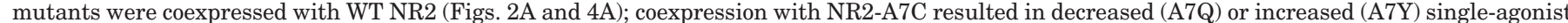

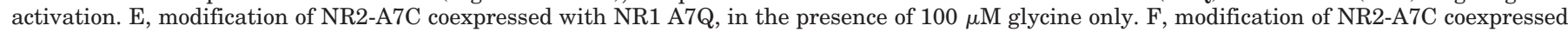

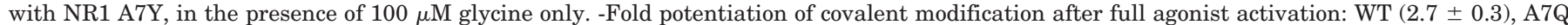

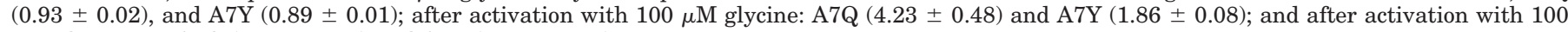
$\mu \mathrm{M}$ glutamate: A7Q $(2.20 \pm 0.27)$ and A7Y $(1.91 \pm 0.22)$. 
gree of potentiation. As expected, we observed that MTSEA modification potentiated currents to approximately the same level, regardless of agonist (Fig. 5D). Thus, modification of NR2A7C potentiates partially activated receptors, whereas after activation with full agonist, modification elicits no additional response from the receptor. This result suggests that our A7 mutants, unlike WT receptors, shift their NR2 M3 domains into a maximally activated conformation after full agonist treatment, consistent with an increased $P_{\mathrm{o}}$.

Interestingly, coexpression of A7Q and A7Y with NR2-A7C altered the percentage of glycine and glutamate-only current observed. A7Q exhibited only $21 \%$ glycine-only and $39 \%$ glutamate-only current, compared with 73 and $71 \%$ when coexpressed with WT NR2. In contrast, the glycine-only current observed in A7Y increased from 49 to 53\%, and glutamateonly current increased from 44 to $57 \%$. These results provide strong evidence for intersubunit interaction at the transmembrane level, but also complicate interpretation of NR2 M3 accessibility. Because our NR1 A7 residues seem to interact with NR2 A7C, and studies have shown that the SYTANLAAF regions of adjacent glutamate receptor subunits move close enough to coordinate cadmium ions (Sobolevsky et al., 2003), movements of the NR1 M3 segment could potentially alter accessibility of NR2-A7C. However, the increased potency seen at the glutamate binding domain, as well as the observed correlation between accessibility and activation, support the conclusion that the NR2 M3 domain in our mutants is shifted to a more activated conformation.

Remarkably, introduction of the A7Q and A7Y substitutions into the NR2 subunit did not result in similar gain-offunction phenotypes (Fig. 6). A left-shifted concentration midpoint was observed in NR2 A7Y $\left(\mathrm{EC}_{50}=0.69 \mu \mathrm{M} ; n=6\right)$, as well as a small degree of glycine-only current (16\%). NR2 $\mathrm{A} 7 \mathrm{Q}$, however, displayed no activation in the absence of glutamate and only a slight decrease in concentration midpoint $\left(\mathrm{EC}_{50}=2.03 \mu \mathrm{M} ; n=5\right)$. This observation is consistent with studies suggesting that the NR1 and NR2 M3 segments contribute unequally to the channel pore (Sobolevsky et al.,

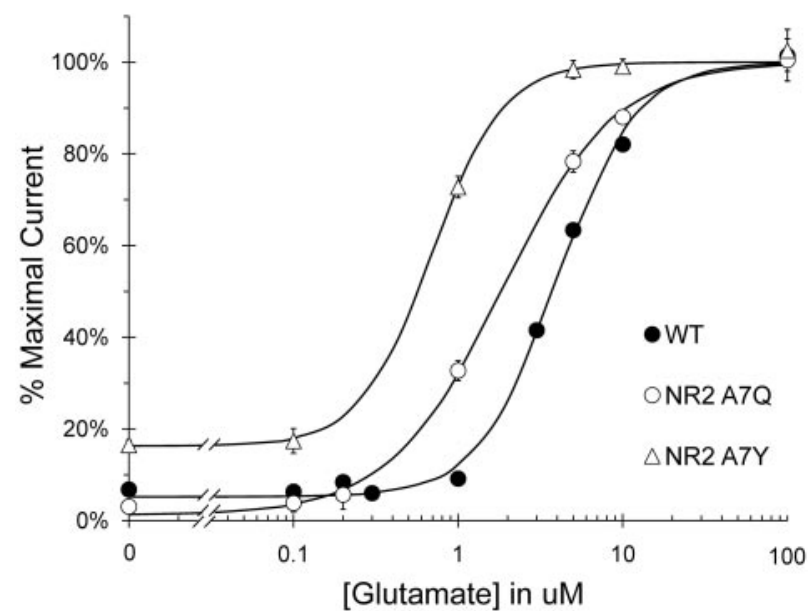

Fig. 6. NR1 gain-of-function phenotypes are not conserved in NR2. Q and $\mathrm{Y}$ substitutions were introduced at the A7 position in the NR2 subunit, and glutamate concentration-response curves were determined in the presence of $100 \mu \mathrm{M}$ glycine. NR2 A7Q exhibited no significant glycine only current ( $3 \pm 1 \%$ ), whereas NR2 A7Y displayed only a small amount $(16 \pm 1 \%$, compared with $7 \pm 1 \%$ for WT). Glutamate concentration midpoints were $2.03 \mu \mathrm{M}(\mathrm{NR} 2 \mathrm{~A} 7 \mathrm{Q})$ and $0.69 \mu \mathrm{M}$ (NR2 A7Y), Hill coefficients were $1.2(\mathrm{NR} 2 \mathrm{~A} 7 \mathrm{Q})$ and 2.0 (NR2 A7Y).
2007) and supports different functional roles for the subunits in channel activation.

A7Q and A7Y Exhibit Altered Sensitivity to Pore Blockers. To determine the effect of our A7 substitutions on gating, we assessed the sensitivity of both mutants to a selection of channel blockers. These antagonists bind to a site distinct from the agonist binding site and thus cannot be displaced by increased agonist concentration. In addition, "use-dependent" channel blockers are only effective on activated channels. MK-801 (dizocilpine), ketamine, memantine, and $\mathrm{Mg}^{2+}$ are all use-dependent antagonists, thought to bind deep in the pore of the NMDA receptor (Chen and Lipton, 2005; Yuan et al., 2005). Initially, we measured inhibition of our A7 mutants with saturating concentrations of each antagonist (Fig. 7A). Both mutants were fully inhibited by all four antagonists, with the exception of ketamine, which was slightly less effective at blocking A7Q. In contrast, lower concentrations of memantine, ketamine, and MK-801 were significantly less effective at inhibiting both mutant channels (Fig. 7B). Most notably, 200 nM MK-801 blocked A7Q receptors by only $9 \%$, despite being well over the wild-type $\mathrm{IC}_{50}$ value of $66 \mathrm{nM}$ (Avenet et al., 1997). Memantine concentration-response curves were also constructed for both mutants, indicating a 20 -fold decrease in memantine $\mathrm{IC}_{50}$ and confirming that channel block still occurs in a concentration-dependent manner (Fig. 7C). Because previous experiments have suggested an increased open probability in these mutants (Fig. 5), open channel blockers would actually be expected to have increased access to their binding sites. Our results with $\mathrm{Mg}^{2+}$ are consistent with this explanation, because $100 \mu \mathrm{M}$ $\mathrm{Mg}^{2+}$ is approximately $30 \%$ more effective at inhibiting A7Q and A7Y than wild-type receptors (Fig. 7B). In contrast, decreased sensitivity to open channel block may indicate disruption of the blocker binding site, such that increased accessibility is counterbalanced by reduced affinity. Modification of the M3 domain has been shown previously to affect sensitivity to organic channel blockers but not $\mathrm{Mg}^{2+}$ inhibition (Yuan et al., 2005); taken together with our data, these findings are consistent with a perturbation of the outer porelining transmembrane domains, with little effect on the $\mathrm{Mg}^{2+}$ binding site (Sharma and Stevens, 1996; Wollmuth et al., 1996).

Reduced Sensitivity to Proton Inhibition. NMDA receptors are highly sensitive to $\mathrm{pH}$ modulation, resulting in tonic proton inhibition of almost $50 \%$ under physiological conditions. Although the proton-sensing domain has yet to be definitively localized, many mutations in the S1-M1 and M3-S2 linker regions, as well as the SYTANLAAF region, exhibit reduced proton sensitivity (Low et al., 2003). In addition, several allosteric modulators, including zinc and ifenprodil, have been proposed to mediate their effects through modulation of proton inhibition (Zheng et al., 2001). To determine the $\mathrm{pH}$ sensitivity of our A7 substitutions, we constructed proton concentration-response curves for both mutants (Fig. 8A). A7Y exhibited decreased proton sensitivity, with a $\mathrm{p} K_{\mathrm{a}}$ value of 6.6 compared with 7.0 for wild-type receptors. A7Q receptors had an even more dramatic phenotype, demonstrating complete resistance to proton inhibition at $\mathrm{pH} 6.0$; instead, these channels were actually inhibited at higher $\mathrm{pH}$. Similar results were seen in A7Y receptors, which exhibit some current inhibition at $\mathrm{pH} 7.5$ and above. Because it is unclear how the tyrosine and glutamine substitutions 
stabilize M3 in an active conformation, and the $\mathrm{p} K_{\mathrm{a}}$ values of these side chains within the local environment are unknown, we cannot speculate on how protons may affect these interactions. However, decreased proton inhibition, seen to vary-
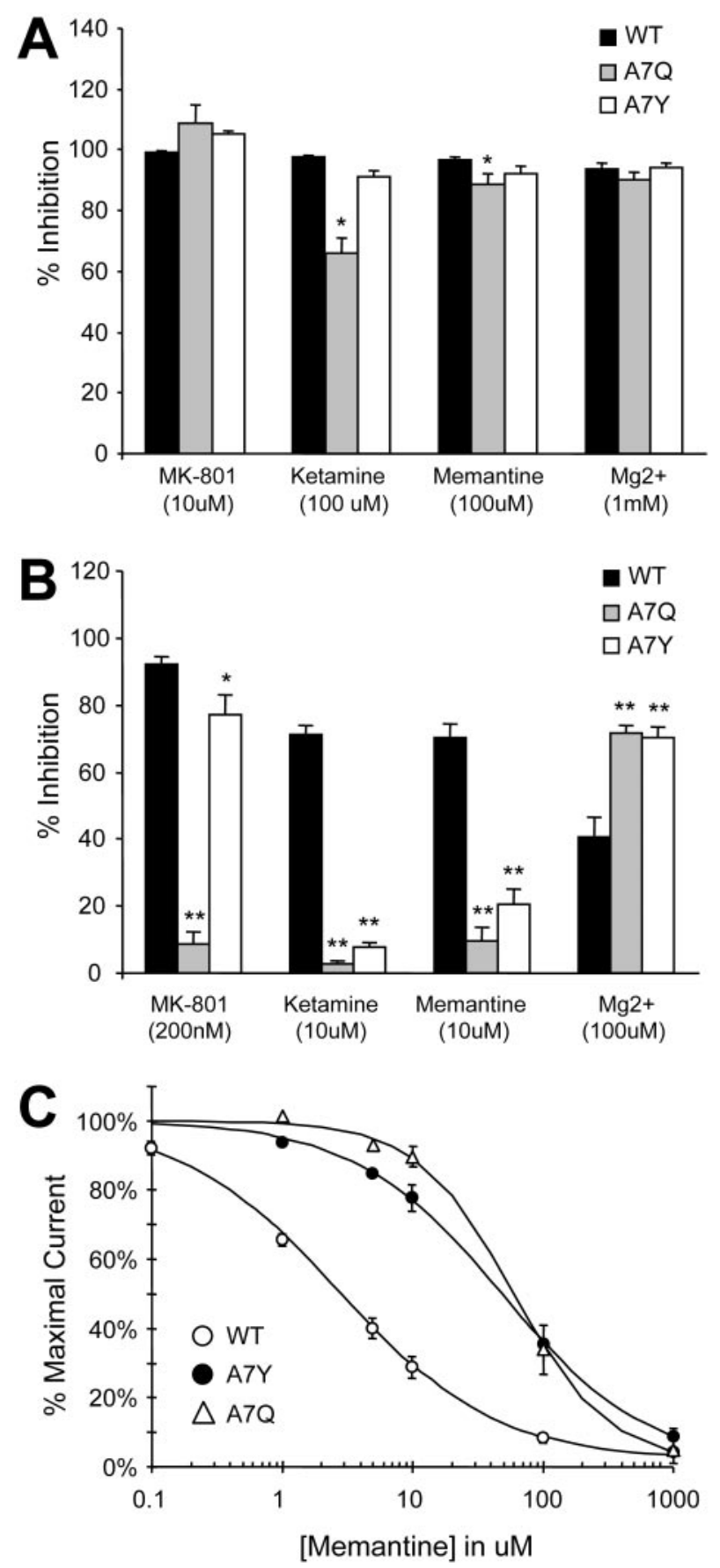

Fig. 7. Decreased sensitivity to pore blockers in A7Q and A7Y. A, Percentage of inhibition by various channel blockers was measured in the presence of $100 \mu \mathrm{M}$ glycine and $100 \mu \mathrm{M}$ glutamate. Inhibition of A7Q and A7Y was similar to WT with all antagonists tested except ketamine, which was less effective at inhibiting A7Q. B, both mutants exhibited decreased sensitivity to lower concentrations of MK-801, ketamine, and memantine, but increased sensitivity to low magnesium. C, memantine concentration-inhibition curves obtained in the presence of $100 \mu \mathrm{M}$ glycine and $100 \mu \mathrm{M}$ glutamate. Both mutants were significantly less sensitive to inhibition by memantine. $\mathrm{IC}_{50}$ values were $59.0 \mu \mathrm{M}$ for $\mathrm{A} 7 \mathrm{Q}$ and $48.3 \mu \mathrm{M}$ for A7Y, compared with $2.67 \mu \mathrm{M}$ for WT. Hill coefficients were 1.2 for A7Q, 0.8 for A7Y and 0.7 for WT. *, $p<0.05$ (significant); **, $p<$ 0.01 (highly significant). ing degrees in both A7 mutants, supports the hypothesis that the proton sensor lies upstream of M3 in the activation pathway. Furthermore, these results are consistent with the suggestion that protons block a pregating conformational change, which may be affected by the altered activation equilibrium in these mutants (Banke et al., 2005).

Activation by a Positive Allosteric Modulator. NMDA receptors are also subject to positive allosteric modulation by PS, an endogenous neurosteroid shown to increase peak channel open probability (Horak et al., 2004). PS binding has been localized to a steroid modulatory domain, SMD1, which is composed of the M4 transmembrane domain and helix $\mathrm{J} / \mathrm{K}$, located within the S2 segment of the ligand binding domain (Jang et al., 2004). Because PS is a charged molecule and potentiation displays no voltage dependence, helix $\mathrm{J} / \mathrm{K}$ is a more likely candidate for direct binding (Park-Chung et al., 1997).

We evaluated the potentiating effects of PS on our mutants using two different protocols: first, PS was applied between full agonist test pulses; and second, receptors were treated with full agonist and $100 \mu \mathrm{M}$ PS concurrently (Fig. 8B). Previous studies have shown weaker potentiation with the second protocol, because activation decreases receptor affinity for PS (Horak et al., 2004). Our results confirmed this trend, because wild-type receptors showed $\sim 20 \%$ less potentiation with concurrent application. Although PS preapplication produced no potentiation in A7Q or A7Y receptors, concurrent application of PS and full agonist potentiated responses in both mutants. Further testing revealed that PS application alone was sufficient to partially activate these mutants; A7Q and A7Y were activated 20 and $35 \%$, respectively, by $100 \mu \mathrm{M}$ PS, whereas wild-type receptors showed no response (Fig. 8C). This result likely accounts for the concurrent potentiation and indicates that the effects of PS treatment are more rapidly reversible in these mutants, because they seem to dissipate while switching solutions. More importantly, the ability of a positive allosteric modulator to partially activate $\mathrm{A} 7 \mathrm{Q}$ and $\mathrm{A} 7 \mathrm{Y}$, even in the absence of agonist, supports our hypothesis of a shifted activation equilibrium.

\section{Discussion}

The highly conserved SYTANLAAF region has previously been implicated in glutamate receptor activation, using both substituted cysteine modification (Jones et al., 2002; Yuan et al., 2005) and introduction of the lurcher mutation (Taverna et al., 2000; Klein and Howe, 2004). In the present study, we have described two gain-of-function mutations at position A7 of NR1, both of which significantly shift the activation equilibrium of the receptor. We further characterized these mutants using antagonists, allosteric modulators, and substituted cysteine modification techniques, resulting in several noteworthy observations: 1) shifting the NR1 M3 to an active conformation stabilizes the glycine binding domain in a closed cleft state, 2) the altered activation equilibrium of the NR1 subunit propagates to the NR2 subunit, and 3) the lurcher-like phenotype exhibited by these mutants seems to arise from an allosteric coupling mechanism. These conclusions, which we have integrated into a model of NMDA receptor activation (Fig. 9), both complement and extend the results of many recent structure-function studies.

Functional Conservation of the M3 Domain. Previous work has demonstrated that the functional role of M3 is 
conserved across NR2 subtypes (Yuan et al., 2005). Strict sequence conservation and the unique phenotype of $\mathrm{A} 7 \mathrm{C}$ suggest that M3 could be functionally conserved between NR1 and NR2 subunits as well; however, NR1 and NR2 contribute differently to the inner pore of the NMDA receptor (Kuner et al., 1996), and studies have suggested that their M3 domains may be staggered by a full $\alpha$-helical turn (Sobolevsky et al., 2002a). Furthermore, recent work evaluating the voltage dependence of cysteine modification has suggested that the NR1 M3 domain contributes mostly to the deep portion of the pore, whereas the NR2 M3 comprises more of the shallow extracellular vestibule (Sobolevsky et al., 2007). The gain-of-function activation phenotype we describe in NR1 A7Q and A7Y, and its absence in NR2, support a structural asymmetry between the subunits. Our results are also consistent with different functional roles, including distinct fast and slow pregating conformational changes medi- ated by NR1 and NR2, respectively (Banke and Traynelis, 2003). This mechanism could allow NR1 substitutions to alter the activation equilibrium of the receptor without rendering it constitutively active. Interestingly, recent work with the inhibitory NR3 subunit has indicated that its M3 domain is relatively rigid and does not undergo activationdependent rearrangement, providing a possible explanation for its "dominant-negative" effect (Wada et al., 2006).

Interaction between the M3 Domains. Currently, there is no crystal structure data to explain how A7Q and A7Y physically alter the conformation of M3, but the similar characteristics observed in other gain-of-function mutants suggest that they may act through a similar mechanism. The

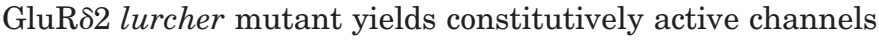
(Zuo et al., 1997), and the same mutation in GluR1 and GluR6 receptors increases agonist potency, slows deactivation, and converts an antagonist into an agonist (Kohda et
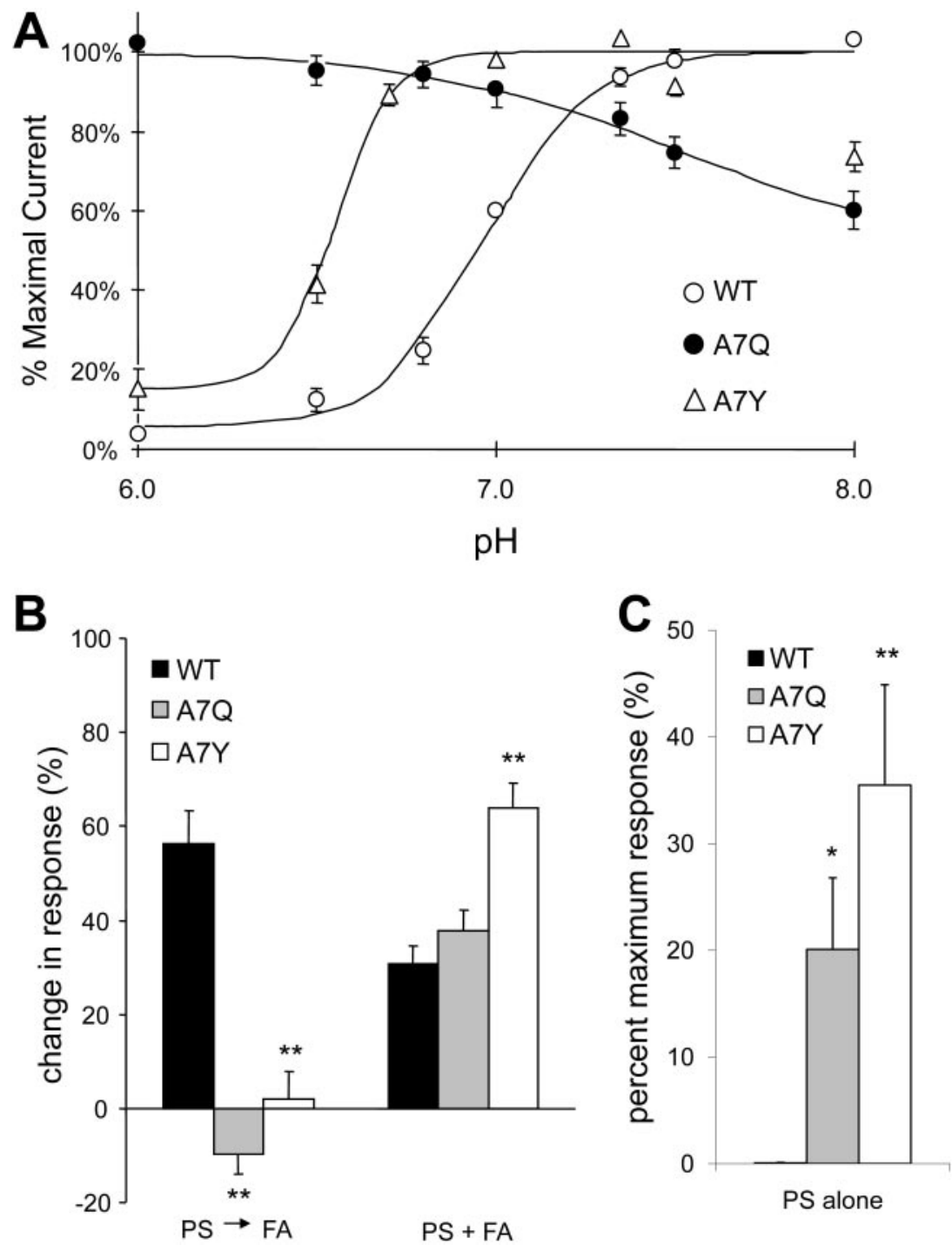

Fig. 8. Altered response of $\mathrm{A} 7 \mathrm{Q}$ and $\mathrm{A} 7 \mathrm{Y}$ to allosteric modulators. A, proton inhibition curves in the presence of $100 \mu \mathrm{M}$ glycine and $100 \mu \mathrm{M}$ glutamate. WT receptors are highly sensitive to $\mathrm{pH}$ modulation, with a $\mathrm{p} K_{\mathrm{a}}$ value of 6.97. Decreased proton inhibition was observed in $\mathrm{A} 7 \mathrm{Y}\left(\mathrm{p} K_{\mathrm{a}}=6.59\right.$ ), whereas A7Q was insensitive to proton block and inhibited by high $\mathrm{pH}$. Inhibition of A7Y was also seen at high pH values, which were not used in calculating the $\mathrm{p} K_{\mathrm{a}}$ for that mutant. B, current potentiation by $100 \mu \mathrm{M}$ PS, applied either before or in the presence of agonist. PS preapplication yielded $\sim 50 \%$ potentiation of the WT full agonist response but had little effect on A7Q or A7Y (WT: $56 \pm 7 \%$; A7Q: $-10 \pm 5 \%$; and A7Y: $2 \pm 6 \%$ ). However, concurrent treatment with PS and full agonist restored potentiation in both mutants (WT: $31 \pm 4 \%$; A7Q: $38 \pm 4 \%$; and A7Y: $64 \pm 5 \%$ ). C, application of $100 \mu \mathrm{M}$ PS was found to partially activate both A7Q and A7Y even in the absence of agonist, whereas having no effect on WT (WT: $0.1 \pm 0.0 \%$; A7Q: $16 \pm 6 \%$; and A7Y: $30 \pm 10 \%$ ). $*, p<0.05$ (significant); ${ }^{* *}, p<0.01$ (highly significant). 
al., 2000; Taverna et al., 2000). Consistent with our own results, the lurcher mutation increases glutamate potency and alters activation kinetics when introduced into NR1, but not NR2 (Kohda et al., 2000; Hu and Zheng, 2005), and it exhibits dominance in heteromeric GluR2 and GluR6 receptors (Schwarz et al., 2001). Furthermore, current potentiation and/or constitutive activity have been observed after modification of several substituted cysteines within the SYTANLAAF region (Beck et al., 1999; Jones et al., 2002; Sobolevsky et al., 2002b, 2003), and the decreased sensitivity to competitive antagonists, pore blockers, and allosteric modulators displayed by our A7 mutants bears a striking resemblance to MTSEA-modified A7C receptors (Yuan et al., 2005). One recent study, in which residues displaying state-dependent accessibility were found to cluster together on helical net diagrams, opposite from putative pore-lining residues, led to the proposal that positions exhibiting lurcher-like effects may be located at dynamic interfaces between transmembrane segments (Sobolevsky et al., 2007).

These results, combined with the reported 2 -fold symmetry of the $\alpha$-amino-3-hydroxy-5-methyl-4-isoxazolepropionic acid receptor extracellular vestibule (Sobolevsky et al., 2004), suggest a mechanism by which certain substituents at position A7 could affect receptor activation (Fig. 9). Gating of glutamate receptors is believed to arise from separation of the linker regions, which results from $\mathrm{LBD}$ closure and requires a stable dimer interface (Furukawa et al., 2005). Because

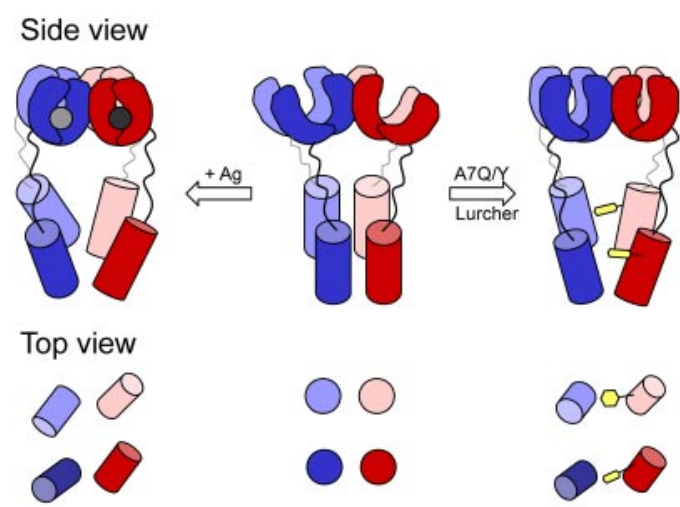

Fig. 9. Proposed structural mechanism for the effect of M3 substitutions on NMDA receptor activation. Middle, an NMDA receptor in its resting state, with LBDs stabilized in the open state by tension from the LBD-M3 linkers. For clarity, only the LBDs, linkers, and C-terminal portion (SYTANLAAF) of the M3 segments are shown. Addition of glycine and glutamate (left), leads to closure of the LBD, and stability of the dimer interface transfers this force via the linkers to the M3 segment. Linker separation pulls the M3 domains into their activated conformations, in which NR1-NR1 and NR2-NR2 M3 pairs rotate and move relative to each other (bottom left). This model incorporates the 2 -fold rotational symmetry previously suggested for the GluR2 outer vestibule (Sobolevsky et al., 2004), the vertical staggering of NR1 and NR2 within the pore (Sobolevsky et al., 2002a), and the heteromeric LBD interface seen in the NR1-NR2A crystal structure (Furukawa et al., 2005). Right, suggested mechanism for the activation phenotypes seen in lurcher-like gain-offunction mutants: introduction of a properly positioned substituent within the NR1 M3 domain could sterically hinder the NR2 M3 from returning to its resting position, thus shifting the activation equilibrium toward an activated state. Therefore, NR1 M3 mutations could cause intersubunit effects, increasing the accessibility of the NR2 M3 and altering the conformation of the outer pore. Relief of linker tension would allow the LBDs to assume a more closed conformation, protecting the cleft from thiol modification. NR1 and NR2 subunits are depicted in red and blue, respectively, and the yellow octagon represents a mutant or modified side chain (such as a glutamine, tyrosine, or covalently modified cysteine).
NMDA receptor LBD dimers are heteromeric, application of this mechanism to a structural model of $\alpha$-amino-3-hydroxy5-methyl-4-isoxazolepropionic acid receptor gating suggests that the NR1 and NR2 SYTANLAAF regions move apart during channel activation (Sobolevsky et al., 2004). Thus, linker separation would lead to separation between the NR1 and NR2 M3 domains, whereas moving homomeric M3 domains closer together. Certain bulky substituents, such as tyrosine or MTSEA, could sterically hinder the NR2 M3 domain from approaching NR1, destabilizing the closed state and shifting the activation equilibrium of the receptor. This mechanism, although speculative, would account for the increased accessibility and altered single-agonist currents observed when NR2-A7C was coexpressed with our mutants, and explain the dominant effect of NR1 mutations on the intact receptor. Thus, the ability of our A7 mutants to be partially activated by either agonist may be due to intersubunit interactions at the transmembrane level.

Coupling of M3 Movement to LBD Closure. The decreased LBD accessibility exhibited by both A7Q and A7Y, which we interpret as stabilization of the closed cleft conformation, provides the first direct structural evidence that the M3 domain can affect LBD cleft closure. In addition, our results present a structural explanation for many previously published observations. Ligand-gated ion channels display higher agonist affinity in the open state, due to thousand-fold slower dissociation rates (Grosman and Auerbach, 2001). Because LBD stability is a major determinant of agonist affinity (Robert et al., 2005), these results could be explained by reverse allosteric coupling between M3 and the LBD. Closed cleft LBD stabilization would also account for results from MTSEA-modified A7C receptors and lurcher mutants, which exhibit increased agonist efficacy, slow deactivation, and decreased sensitivity to competitive antagonists (Kohda et al., 2000; Jones et al., 2002; Yuan et al., 2005). Finally, this mechanism provides a structural context for the trapping effect of 9-aminoacridine (9-AA), a sequential or "foot-in-thedoor" blocker. Upon binding to the pore, 9-AA prevents channel closure, leading to an open-blocked state from which glycine and glutamate cannot dissociate (Benveniste and Mayer, 1995). According to our hypothesis, the presence of 9-AA in the pore precludes M3 returning to its inactive position, thus maintaining the LBDs in their closed cleft conformations.

Additional evidence in support of this theory can be found in molecular dynamics and crystal structure data. Ligandfree closed cleft conformations have been observed in the structures of several homologous LBDs, including GluR0 (Mayer et al., 2001), mGluR1 (Kunishima et al., 2000), and the glucose/galactose receptor (Flocco and Mowbray, 1994). Glutamate-free molecular dynamics simulations of the GluR2 LBD showed no domain reopening for the lifetime of the simulation (Arinaminpathy et al., 2002). Furthermore, the periplasmic histidine-binding protein rapidly fluctuates between its open and closed conformations in the absence of ligand (Wolf et al., 1994). Thus, closed apo states of the LBD are readily observed in the absence of attached transmembrane domains, raising the possibility that the LBD-TM linkers are responsible for stabilizing the LBDs in the open cleft state. Altering the conformation of M3 could relieve this tension, allowing the glycine binding cleft to behave more like its soluble homolog. 
Our results demonstrate that the glycine binding cleft is inaccessible to modification, indicating a significant degree of cleft closure; however, the resulting conformation may not correspond to a full agonist-bound state, particularly because both mutants are activated further by additional glycine. And unlike GluR2, NR1 does not exhibit a correlation between cleft closure and agonist efficacy, so additional structural determinants are necessary to induce full channel activation (Inanobe et al., 2005).

Allosteric Coupling in lurcher Mutants. A recent article reported the generation of a lurcher phenotype by replacing the GluR1 S1-M1 linker with the corresponding GluR $\delta 2$ region, raising the question of whether these gain-of-function properties result from allosteric coupling or uncoupling between the LBD and transmembrane region (Schmid et al., 2007). Both mechanisms are thought to describe the GluR1 linker chimera, because a portion of the observed constitutive current was insensitive to open channel blockers. A7Q and A7Y, however, were fully inhibited by open channel blockers, and no constitutive activation was observed in the absence of both agonists. Furthermore, given the altered accessibility of the glycine binding site, PS-induced activation, and decreased (but not abolished) inhibition by competitive antagonists, our data are consistent with an allosteric coupling mechanism. The phenotypes observed in the GluR1 linker chimeras indicate that these linker regions are likely to play a critical role in this mechanism.

Our data suggest that the observed allosteric coupling is mediated by intersubunit interactions, most likely within the transmembrane region; therefore, these contact points represent key regulatory domains controlling NMDA receptor activation, as well as possible therapeutic targets. Our results with antagonists and allosteric modulators also illustrate a point with broad applicability to receptor pharmacology: gain-of-function mutations such as lurcher can alter blocker potency and/or cause structural alterations of blocker sites, even at relatively long range. In addition, such mutants may be unresponsive to regulation by endogenous modulators, exacerbating the physiological phenotype.

\section{Acknowledgments}

We thank Dr. Timothy Haystead for assistance with the LC-MS experiments and Dr. Kevin Jones for critical evaluation of the manuscript.

\section{References}

Arinaminpathy Y, Sansom MSP, and Biggin PC (2002) Molecular dynamics simulations of the ligand-binding domain of the ionotropic glutamate receptor GluR2. Biophys $J$ 82:676-683.

Auerbach A and Zhou Y (2005) Gating reaction mechanisms for NMDA receptor channels. J Neurosci 25:7914-7923.

Avenet P, Leonardon J, Besnard F, Graham D, Depoortere H, and Scatton B (1997) Antagonist properties of eliprodil and other NMDA receptor antagonists at rat NR1A/NR2A and NR1A/NR2B receptors expressed in Xenopus oocytes. Neurosci Lett 223:133-136.

Banke TG and Traynelis SF (2003) Activation of NR1/NR2B NMDA receptors. Nat Neurosci 6:144-152.

Banke TG, Dravid SM, and Traynelis SF (2005) Protons trap NR1/NR2B NMDA receptors in a nonconducting state. J Neurosci 25:42-51.

Beck C, Wollmuth LP, Seeburg PH, Sakmann B, and Kuner T (1999) NMDAR channel segments forming the extracellular vestibule inferred from the accessibility of substituted cysteines. Neuron 22:559-570.

Benveniste M and Mayer ML (1995) Trapping of glutamate and glycine during open channel block of rat hippocampal neuron NMDA receptors by 9 -aminoacridine. J Physiol 483:367-384.

Chen H-SV and Lipton SA (2005) Pharmacological implications of two distinct mechanisms of interaction of memantine with $N$-methyl-D-aspartate-gated channels. J Pharmacol Exp Ther 314:961-971.

Chen PE, Geballe MT, Stansfeld PJ, Johnston AR, Yuan H, Jacob AL, Snyder JP,
Traynelis SF, and Wyllie DJA (2005) Structural features of the glutamate binding site in recombinant NR1/NR2A $N$-methyl-D-aspartate receptors determined by site-directed mutagenesis and molecular modeling. Mol Pharmacol 67:1470-1484. Collingridge GL and Bliss TVP (1995) Memories of NMDA receptors and LTP. Trends Neurosci 18:54-56.

Dingledine R, Borges K, Bowie D, and Traynelis SF (1999) The glutamate receptor ion channels. Pharmacol Rev 51:7-62.

Emamian ES, Karayiorgou M, and Gogos JA (2004) Decreased phosphorylation of NMDA receptor type 1 at serine 897 in brains of patients with schizophrenia. $J$ Neurosci 24:1561-1564.

Flocco MM and Mowbray SL (1994) The 1.9 A x-ray structure of a closed unliganded form of the periplasmic glucose/galactose receptor from Salmonella typhimurium. J Biol Chem 269:8931-8936.

Furukawa H and Gouaux E (2003) Mechanisms of activation, inhibition and specificity: crystal structures of the NMDA receptor NR1 ligand-binding core. EMBO J 22:2873-2885.

Furukawa H, Singh SK, Mancusso R, and Gouaux E (2005) Subunit arrangement and function in NMDA receptors. Nature 438:185-192.

Grosman C and Auerbach A (2001) The dissociation of acetylcholine from open nicotinic receptor channels. Proc Natl Acad Sci U S A 98:14102-14107.

Horak M, Vlcek K, Petrovic M, Chodounska H, and Vyklicky L Jr (2004) Molecular mechanism of pregnenolone sulfate action at NR1/NR2B receptors. $J$ Neurosci 24:10318-10325.

$\mathrm{Hu} \mathrm{B}$ and Zheng F (2005) Differential effects on current kinetics by point mutations in the lurcher motif of NR1/NR2A receptors. J Pharmacol Exp Ther 312:899-904. Hynd MR, Scott HL, and Dodd PR (2004) Differential expression of $N$-methyl-Daspartate receptor NR2 isoforms in Alzheimer's disease. J Neurochem 90:913-919.

Inanobe A, Furukawa H, and Gouaux E (2005) Mechanism of partial agonist action at the NR1 subunit of NMDA receptors. Neuron 47:71-84.

Jang M-K, Mierke DF, Russek SJ, and Farb DH (2004) A steroid modulatory domain on NR2B controls $N$-methyl-D-aspartate receptor proton sensitivity. Proc Nat Acad Sci U S A 101:8198-8203.

Johnson JW and Ascher P (1987) Glycine potentiates the NMDA response in cultured mouse brain neurons. Nature 325:529-531.

Jones KS, VanDongen HMA, and VanDongen AMJ (2002) The NMDA receptor M3 segment is a conserved transduction element coupling ligand binding to channel opening. J Neurosci 22:2044-2053.

Kalbaugh TL, VanDongen HMA, and VanDongen AMJ (2004) Ligand-binding residues integrate affinity and efficacy in the NMDA receptor. Mol Pharmacol 66 209-219.

Kleckner NW and Dingledine R (1988) Requirement for glycine in activation of NMDA-receptors expressed in Xenopus oocytes. Science 241:835-837.

Klein RM and Howe JR (2004) Effects of the Lurcher mutation on GluR1 desensitization and activation kinetics. J Neurosci 24:4941-4951.

Kohda K, Wang Y, and Yuzaki M (2000) Mutation of a glutamate receptor motif reveals its role in gating and [delta]2 receptor channel properties. Nat Neurosci 3:315-322.

Kuner T, Wollmuth LP, Karlin A, Seeburg PH, and Sakmann B (1996) Structure of the NMDA receptor channel M2 segment inferred from the accessibility of substituted cysteines. Neuron 17:343-352.

Kunishima N, Shimada Y, Tsuji Y, Sato T, Yamamoto M, Kumasaka T, Nakanishi S, Jingami H, and Morikawa K (2000) Structural basis of glutamate recognition by a dimeric metabotropic glutamate receptor. Nature 407:971-977.

Leonard JP and Kelso SR (1990) Apparent desensitization of NMDA responses in xenopus oocytes involves calcium-dependent chloride current. Neuron 4:53-60.

Lerma J, Zukin RS, and Bennett MVL (1990) Glycine decreases desensitization of $N$-methyl-D-aspartate (NMDA) receptors expressed in Xenopus oocytes and is required for NMDA responses. Proc Natl Acad Sci U S A 87:2354-2358.

Li JZ and Lester HA (1997) Cyclic nucleotide-gated channels: structural basis of ligand efficacy and allosteric modulation. Q Rev Biophys 30:177-193.

Low C-M, Zheng F, Lyuboslavsky P, and Traynelis SF (2000) Molecular determinants of coordinated proton and zinc inhibition of $N$-methyl-D-aspartate NR1/ NR2A receptors. Proc Natl Acad Sci U S A 97:11062-11067.

Low C-M, Lyuboslavsky P, French A, Le P, Wyatte K, Thiel WH, Marchan EM, Igarashi K, Kashiwagi K, Gernert K, et al. (2003) Molecular determinants of proton-sensitive $N$-methyl-D-aspartate receptor gating. Mol Pharmacol 63:12121222.

Malenka RC and Nicoll RA (1999) Long-term potentiation-a decade of progress? Science 285:1870-1874.

Mayer ML (2006) Glutamate receptors at atomic resolution. Nature 440:456-462.

Mayer ML, Westbrook GL, and Guthrie PB (1984) Voltage-dependent block by $\mathrm{Mg}^{2+}$ of NMDA responses in spinal cord neurones. Nature 309:261-263.

Mayer ML, Olson R, and Gouaux E (2001) Mechanisms for ligand binding to GluR0 ion channels: crystal structures of the glutamate and serine complexes and a closed apo state. J Mol Biol 311:815-836.

Mody I and MacDonald JF (1995) NMDA receptor-dependent excitotoxicity: the role of intracellular $\mathrm{Ca}^{2+}$ release. Trends Pharmacol Sci 16:356-359.

Muir KW (2006) Glutamate-based therapeutic approaches: clinical trials with NMDA antagonists. Curr Opin Pharmacol 6:53-60.

Paoletti P, Ascher P, and Neyton J (1997) High-affinity zinc inhibition of NMDA NR1-NR2A receptors. J Neurosci 17:5711-5725.

Paoletti P, Perin-Dureau F, Fayyazuddin A, Le Goff A, Callebaut I, and Neyton J (2000) Molecular organization of a zinc binding N-terminal modulatory domain in a NMDA receptor subunit. Neuron 28:911-925.

Park-Chung M, Wu F-S, Purdy RH, Malayev AA, Gibbs TT, and Farb DH (1997) Distinct sites for inverse modulation of $N$-methyl-D-aspartate receptors by sulfated steroids. Mol Pharmacol 52:1113-1123.

Paudice P, Gemignani A, and Raiteri M (1998) Evidence for functional native NMDA receptors activated by glycine or serine alone in the absence of glutamatergic coagonist. Eur J Neurosci 10:2934-2944 
Regalado MP, Villarroel A, and Lerma J (2001) Intersubunit cooperativity in the NMDA receptor. Neuron 32:1085-1096.

Robert A, Armstrong N, Gouaux JE, and Howe JR (2005) AMPA receptor binding cleft mutations that alter affinity, efficacy, and recovery from desensitization. J Neurosci 25:3752-3762.

Schmid SM, Korber C, Herrmann S, Werner M, and Hollmann M (2007) A domain linking the AMPA receptor agonist binding site to the ion pore controls gating and causes lurcher properties when mutated. J Neurosci 27:12230-12241.

Schorge S and Colquhoun D (2003) Studies of NMDA receptor function and stoichiometry with truncated and tandem subunits. J Neurosci 23:1151-1158.

Schwarz MK, Pawlak V, Osten P, Mack V, Seeburg PH, and Kohr G (2001) Dominance of the lurcher mutation in heteromeric kainate and AMPA receptor channels. Eur J Neurosci 14:861-868.

Sharma G and Stevens CF (1996) A mutation that alters magnesium block of $N$-methyl-D-aspartate receptor channels. Proc Natl Acad Sci U S A 93:9259-9263.

Sobolevsky AI, Rooney L, and Wollmuth LP (2002a) Staggering of subunits in NMDAR channels. Biophys J 83:3304-3314.

Sobolevsky AI, Beck C, and Wollmuth LP (2002b) Molecular rearrangements of the extracellular vestibule in NMDAR channels during gating. Neuron 33:75-85.

Sobolevsky AI, Yelshansky MV, and Wollmuth LP (2003) Different gating mechanisms in glutamate receptor and $\mathrm{K}^{+}$channels. J Neurosci 23:7559-7568.

Sobolevsky AI, Yelshansky MV, and Wollmuth LP (2004) The outer pore of the glutamate receptor channel has 2-fold rotational symmetry. Neuron 41:367-378.

Sobolevsky AI, Prodromou ML, Yelshansky MV, and Wollmuth LP (2007) Subunitspecific contribution of pore-forming domains to NMDA receptor channel structure and gating. J Gen Physiol 129:509-525.

Stoll L, Hall J, Van Buren N, Hall A, Knight L, Morgan A, Zuger S, Van Deusen H, and Gentile L (2007) Differential regulation of ionotropic glutamate receptors. Biophys J 92:1343-1349.

Taverna F, Xiong Z-G, Brandes L, Roder JC, Salter MW, and MacDonald JF (2000 The lurcher mutation of an $\alpha$-amino-3-hydroxy-5-methyl-4-isoxazolepropionic acid receptor subunit enhances potency of glutamate and converts an antagonist to an agonist. J Biol Chem 275:8475-8479.

Wada A, Takahashi H, Lipton SA, and Chen HSV (2006) NR3A modulates the outer vestibule of the "NMDA" receptor channel. J Neurosci 26:13156-13166.
Weeber EJ and Sweatt JD (2002) Molecular neurobiology of human cognition. Neuron 33:845-848.

Wolf A, Shaw EW, Nikaido K, and Ames GF (1994) The histidine-binding protein undergoes conformational changes in the absence of ligand as analyzed with conformation-specific monoclonal antibodies. J Biol Chem 269:23051-23058.

Wollmuth LP, Kuner T, Seeburg PH, and Sakmann B (1996) Differential contribution of the NR1- and NR2A-subunits to the selectivity filter of recombinant NMDA receptor channels. J Physiol 491:779-797.

Wood MW, VanDongen HMA, and VanDongen AMJ (1995) Structural conservation of ion conduction pathways in $\mathrm{K}$ channels and glutamate receptors. Proc Natl Acad Sci U S A 92:4882-4886.

Wood MW, VanDongen HMA, and VanDongen AMJ (1996) The $5^{\prime}$-untranslated region of the $N$-methyl-D-aspartate receptor NR2A subunit controls efficiency of translation. J Biol Chem 271:8115-8120.

Wood MW, VanDongen HMA, and VanDongen AMJ (1997) An alanine residue in the M3-M4 linker lines the glycine binding pocket of the $N$-methyl-D-aspartate receptor. J Biol Chem 272:3532-3537.

Yuan H, Erreger K, Dravid SM, and Traynelis SF (2005) Conserved structural and functional control of $N$-methyl-D-aspartate receptor gating by transmembrane domain M3. J Biol Chem 280:29708-29716.

Zhang L, Peoples RW, Oz M, Harvey-White J, Weight FF, and Brauneis U (1997) Potentiation of NMDA receptor-mediated responses by dynorphin at low extracellular glycine concentrations. J Neurophysiol 78:582-590.

Zheng F, Erreger K, Low CM, Banke T, Lee CJ, Conn PJ, and Traynelis SF (2001) Allosteric interaction between the amino terminal domain and the ligand binding domain of NR2A. Nat Neurosci 4:894-901.

Zuo J, De Jager PL, Takahashi KA, Jiang W, Linden DJ, and Heintz N (1997) Neurodegeneration in Lurcher mice caused by mutation in [delta]2 glutamate receptor gene. Nature 388:769-773.

Address correspondence to: Dr. Antonius M. J. VanDongen, Duke-NUS Graduate Medical School, 2 Jalan Bukit Merah, Singapore 169547, Singapore. E-mail vando005@mc.duke.edu 


\section{Correction to "The NR1 M3 Domain Mediates Allosteric Coupling in the $N$-Methyl-D-aspartate Receptor"}

In the above article [Blanke ML and VanDongen AM (2008) Mol Pharmacol 74:454465], incorrect figures were printed. The correct figures appear below.

The online version of this article has been corrected in departure from the print version.

The printer regrets this error and apologizes for any confusion or inconvenience it may have caused.

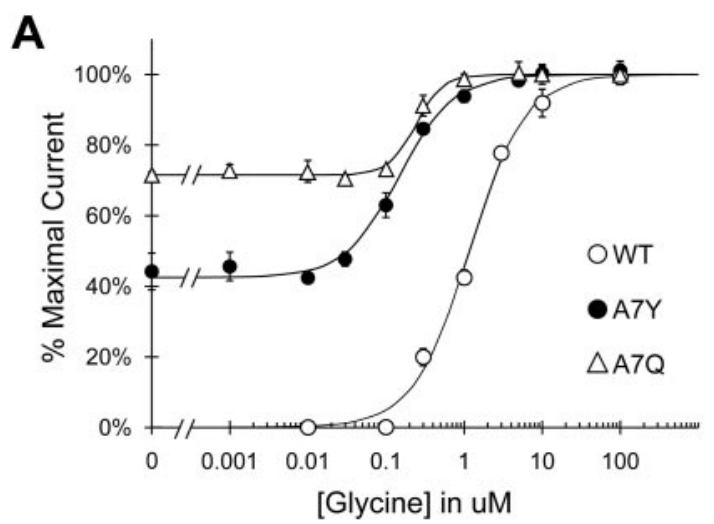

B

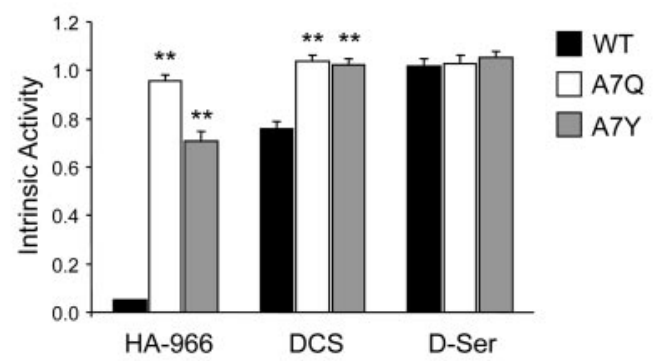

C

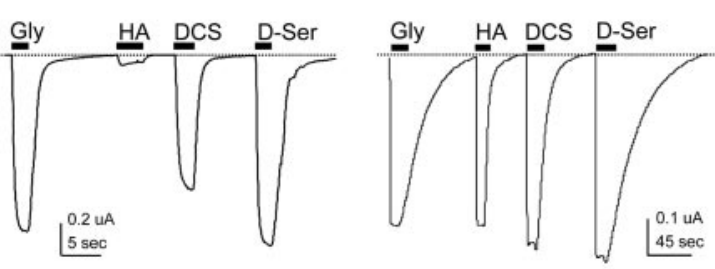

D

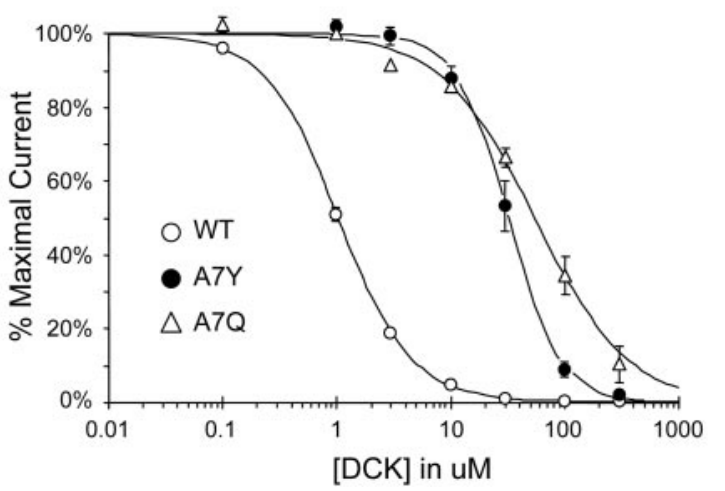

Fig. 2. Altered activation equilibrium in NR1 A7Q and A7Y. A, glycine concentration-response curves in the presence of $100 \mu \mathrm{M}$ L-glutamate. Both A7Q $(\triangle)$ and A7Y $(\bullet)$ exhibited significant glycine-independent current (71 and $44 \%$, respectively) and increased sensitivity to glycine. Concentration midpoints were $0.24 \mu \mathrm{M}$ for $\mathrm{A} 7 \mathrm{Q}$ and $0.15 \mu \mathrm{M}$ for $\mathrm{A} 7 \mathrm{Y}$, compared with $1.16 \mu \mathrm{M}$ for WT. Hill coefficients were 2.5 for A7Q and 1.2 for A7Y, compared with 1.2 for WT. B, response to glycine-site partial agonists, calculated as a fraction of full agonist response. HA-966 (500 $\mu \mathrm{M}), 1 \mathrm{mM}$ DCS, and $100 \mu \mathrm{M}$ D-serine were coapplied with $100 \mu \mathrm{M}$ glutamate. Both $\mathrm{A} 7 \mathrm{Q}$ and $\mathrm{A} 7 \mathrm{Y}$ displayed increased activity in response to HA-966 and DCS, but not D-serine. Intrinsic activities for WT, A7Q, and A7Y were as follows: HA-966: $0.05 \pm 0.00,0.96 \pm 0.02$, and $0.71 \pm 0.04$; DCS: $0.76 \pm 0.03,1.04 \pm 0.02$, and $1.02 \pm 0.02$; and D-Ser: $1.02 \pm 0.03$, $1.03 \pm 0.07$, and $1.06 \pm 0.03$. C, representative whole cell traces illustrating the response of WT (left) and A7Q (right) to glycine-site partial agonists in the presence of $100 \mu \mathrm{M}$ glutamate. A7Q receptors exhibit increased partial agonist responses and markedly slow deactivation. D, inhibition by DCK, a competitive glycine-site antagonist, in the presence of $10 \mu \mathrm{M}$ glycine and $100 \mu \mathrm{M}$ glutamate. Both mutants were significantly less sensitive to DCK inhibition. $\mathrm{IC}_{50}$ values were $51.5 \mu \mathrm{M}$ for $\mathrm{A} 7 \mathrm{Q}$ and $31.7 \mu \mathrm{M}$ for A7Y, compared with $1.04 \mu \mathrm{M}$ for WT. Hill coefficients were 1.1 for A7Q, 2.3 for A7Y, and 1.5 for WT. Calculated $K_{\mathrm{D}}$ values were as follows: A7Q (1.21), A7Y (0.47), and WT (0.11). *, $p<0.05$ (significant); $*_{*}, p<0.01$ (highly significant). 

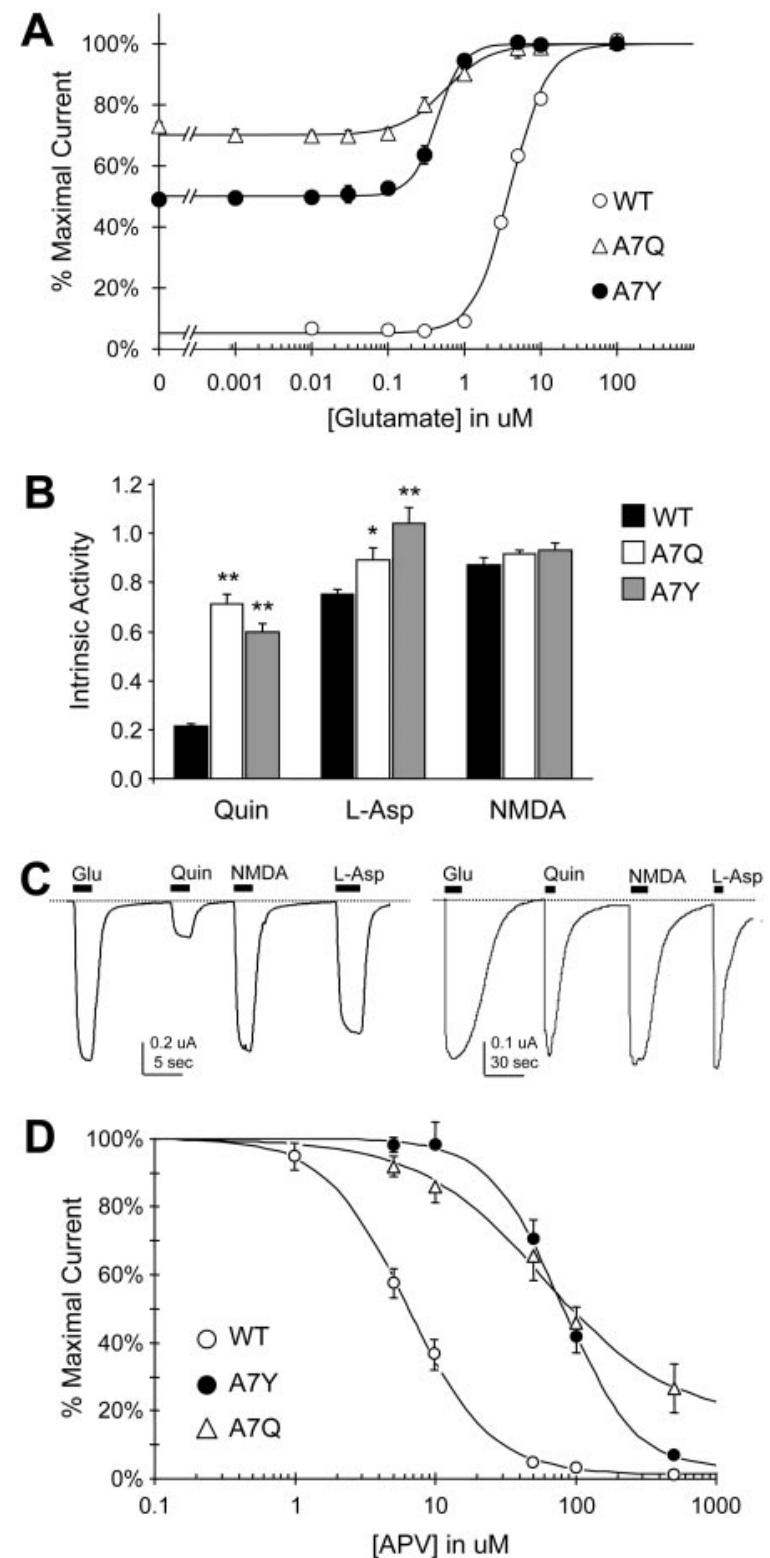

Fig. 4. A7Q and A7Y affect the NR2 ligand binding domain. A, glutamate concentration-response curves in the presence of $100 \mu \mathrm{M}$ glycine. Both A7Q $(\triangle)$ and A7Y $(\bullet)$ exhibited significant glutamate-independent current (73 and $49 \%$, respectively) and increased sensitivity to glutamate. Concentration midpoints were $0.57 \mu \mathrm{M}$ for $\mathrm{A} 7 \mathrm{Q}$ and $0.45 \mu \mathrm{M}$ for $\mathrm{A} 7 \mathrm{Y}$, compared with $4.05 \mu \mathrm{M}$ for WT. Hill coefficients were 1.5 for A7Q, 2.6 for A7Y, and 1.9 for WT. B, response to glutamate-site partial agonists, calculated as a fraction of full agonist response. Quinolinic acid $(10 \mathrm{mM}$; Quin), $1 \mathrm{mM}$ NMDA, and $100 \mu \mathrm{M}$ L-aspartate (L-Asp) were coapplied with $100 \mu \mathrm{M}$ glycine. Both mutants displayed increased activity in response to quinolinic acid and L-aspartate, but not NMDA. Intrinsic activities for WT, A7Q, and A7Y were as follows: Quin: $0.22 \pm 0.01,0.71 \pm 0.04$, and $0.60 \pm 0.03$; L-Asp: $0.75 \pm 0.02,0.89 \pm 0.05$, and $1.04 \pm 0.06$; and NMDA: $0.87 \pm 0.03,0.91 \pm 0.02$, and $0.93 \pm 0.03$. C , representative whole cell traces illustrating the response of WT (left) and A7Q (right) to glutamatesite partial agonists in the presence of $100 \mu \mathrm{M}$ glycine. D, inhibition by APV, a competitive glutamate-site antagonist, in the presence of $100 \mu \mathrm{M}$ glycine and $10 \mu \mathrm{M}$ glutamate. Both mutants were significantly less sensitive to APV inhibition. $\mathrm{IC}_{50}$ values were $86.4 \mu \mathrm{M}$ for A7Q and 83.5 $\mu \mathrm{M}$ for A7Y, compared with $6.22 \mu \mathrm{M}$ for WT. Hill coefficients were 0.8 for $\mathrm{A} 7 \mathrm{Q}, 2.1$ for A7Y, and 1.3 for WT. Calculated $K_{\mathrm{D}}$ values were as follows: A7Q (4.66), A7Y (3.60), and WT (1.79). *, $p<0.05$ (significant); **, $p<$ 0.01 (highly significant). 
A

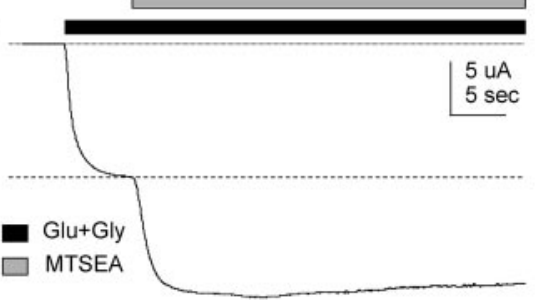

$N R 1+N R 2 A-A 7 C$

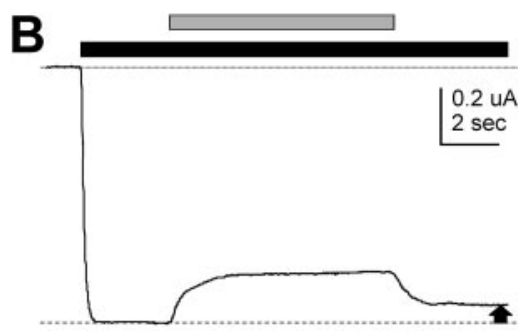

NR1-A7Q + NR2A-A7C

Glutamate + Glycine

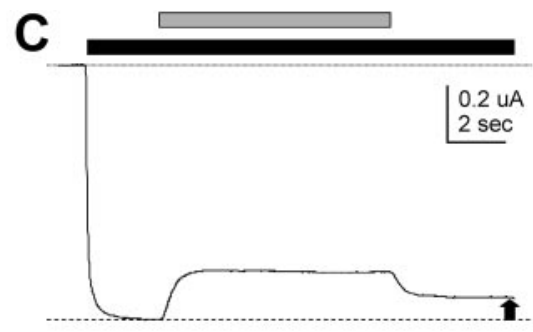

NR1-A7Y + NR2A-A7C

Glutamate + Glycine
D

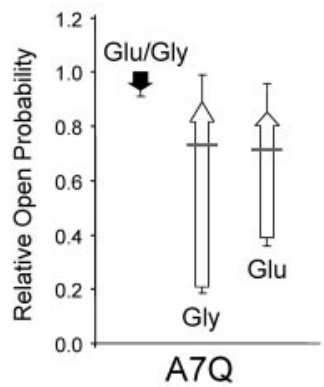

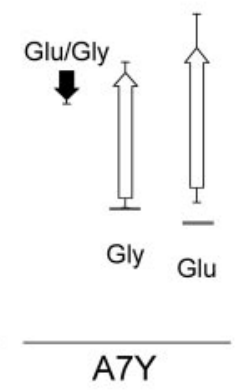

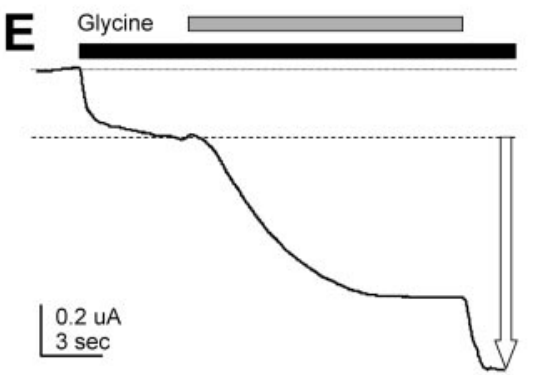

NR1-A7Q + NR2A-A7C

No Glutamate

Fig. 5. A7Q and A7Y increase accessibility of the NR2 M3 domain. NR1 subunits were coexpressed with NR2-A7C, an activation-dependent reporter of M3 accessibility. A, representative whole cell trace showing modification of the NR2-A7C reporter by $0.5 \mathrm{mM}$ MTSEA, a thiol-modifying reagent, in the presence of $100 \mu \mathrm{M}$ glycine and $100 \mu \mathrm{M}$ glutamate. MTSEA modifies and potentiates A7C-containing receptors only in the presence of agonist, increasing channel $P_{\mathrm{o}}$ to approximately 1.0. B, modification of NR2-A7C coexpressed with NR1 A7Q produced only a small amount of irreversible inhibition (filled arrow). Transient inhibition reflects open channel block by the positively charged MTSEA. C, modification of NR2-A7C coexpressed with NR1 A7Y, showing a small inhibition similar to the A7Q response. D, bar graph illustrating the correlation between channel activation and MTSEA modification. The $y$-axis represents relative $P_{o}$, with the maximal response to full agonist assigned a value of 1.0 in each mutant. Inhibition and potentiation are depicted with closed and open arrows, respectively. Gray bars denote the percentage of single-agonist current observed when the mutants were coexpressed with WT NR2 (Figs. 2A and 4A); coexpression with NR2-A7C resulted in decreased (A7Q) or increased (A7Y) single-agonist activation. E, modification of NR2-A7C coexpressed with NR1 A7Q, in the presence of $100 \mu \mathrm{M}$ glycine only. F, modification of NR2-A7C coexpressed with NR1 A7Y, in the presence of $100 \mu \mathrm{M}$ glycine only. -Fold potentiation of covalent modification after full agonist activation: WT (2.7 \pm 0.3$)$, A7Q $(0.93 \pm 0.02)$, and A7Y $(0.89 \pm 0.01)$; after activation with $100 \mu \mathrm{M}$ glycine: A7Q $(4.23 \pm 0.48)$ and A7Y $(1.86 \pm 0.08)$; and after activation with 100 $\mu \mathrm{M}$ glutamate: $\mathrm{A} 7 \mathrm{Q}(2.20 \pm 0.27)$ and $\mathrm{A} 7 \mathrm{Y}(1.91 \pm 0.22)$.

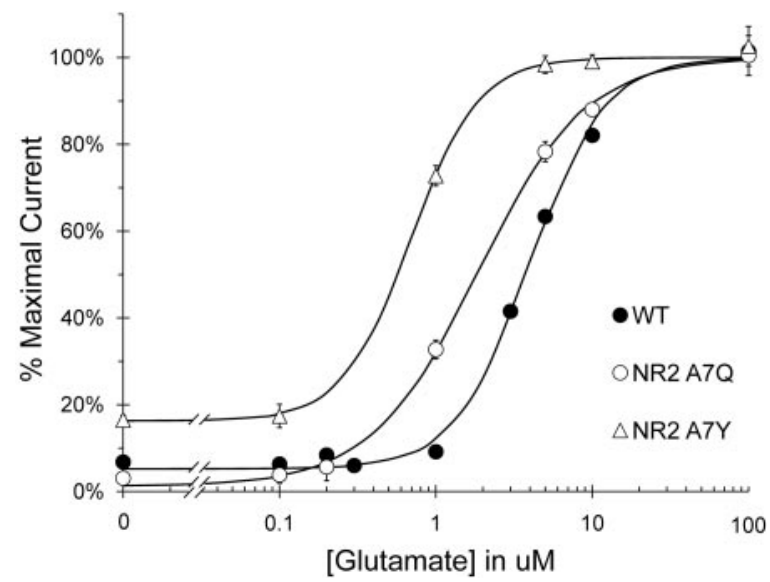

Fig. 6. NR1 gain-of-function phenotypes are not conserved in NR2. Q and Y substitutions were introduced at the A7 position in the NR2 subunit, and glutamate concentration-response curves were determined in the presence of $100 \mu \mathrm{M}$ glycine. NR2 A7Q exhibited no significant glycine only current $(3 \pm 1 \%)$, whereas NR2 A7Y displayed only a small amount $(16 \pm 1 \%$, compared with $7 \pm 1 \%$ for WT). Glutamate concentration midpoints were $2.03 \mu \mathrm{M}$ (NR2 A7Q) and $0.69 \mu \mathrm{M}$ (NR2 A7Y), Hill coefficients were $1.2(\mathrm{NR} 2 \mathrm{~A} 7 \mathrm{Q})$ and $2.0(\mathrm{NR} 2 \mathrm{~A} 7 \mathrm{Y})$. 

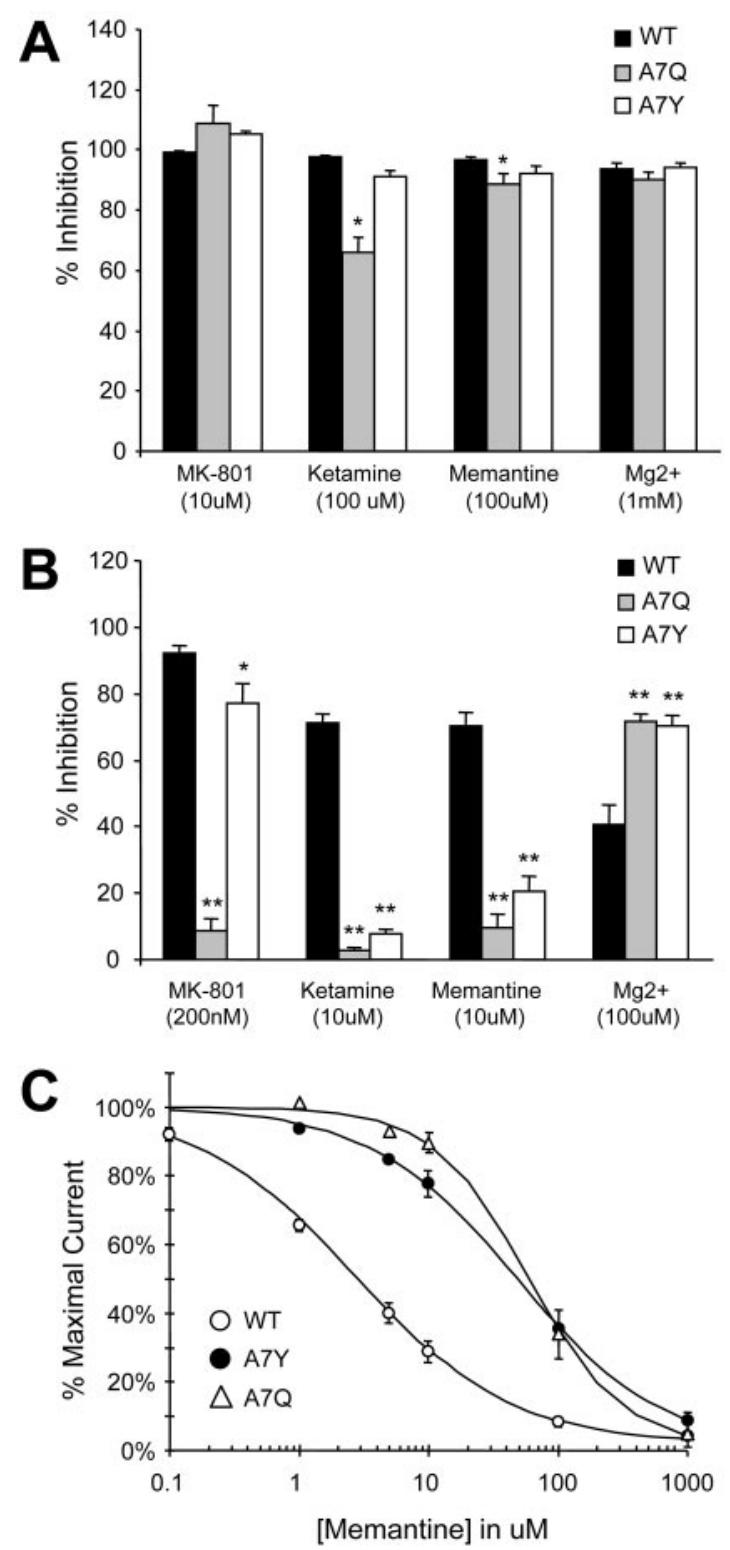

Fig. 7. Decreased sensitivity to pore blockers in A7Q and A7Y. A, Percentage of inhibition by various channel blockers was measured in the presence of $100 \mu \mathrm{M}$ glycine and $100 \mu \mathrm{M}$ glutamate. Inhibition of $\mathrm{A} 7 \mathrm{Q}$ and A7Y was similar to WT with all antagonists tested except ketamine, which was less effective at inhibiting A7Q. B, both mutants exhibited decreased sensitivity to lower concentrations of MK-801, ketamine, and memantine, but increased sensitivity to low magnesium. C, memantine concentration-inhibition curves obtained in the presence of $100 \mu \mathrm{M}$ glycine and $100 \mu \mathrm{M}$ glutamate. Both mutants were significantly less sensitive to inhibition by memantine. $\mathrm{IC}_{50}$ values were $59.0 \mu \mathrm{M}$ for $\mathrm{A} 7 \mathrm{Q}$ and $48.3 \mu \mathrm{M}$ for A7Y, compared with $2.67 \mu \mathrm{M}$ for WT. Hill coefficients were 1.2 for A7Q, 0.8 for A7Y and 0.7 for WT. $*, p<0.05$ (significant); **, $p<$ 0.01 (highly significant).
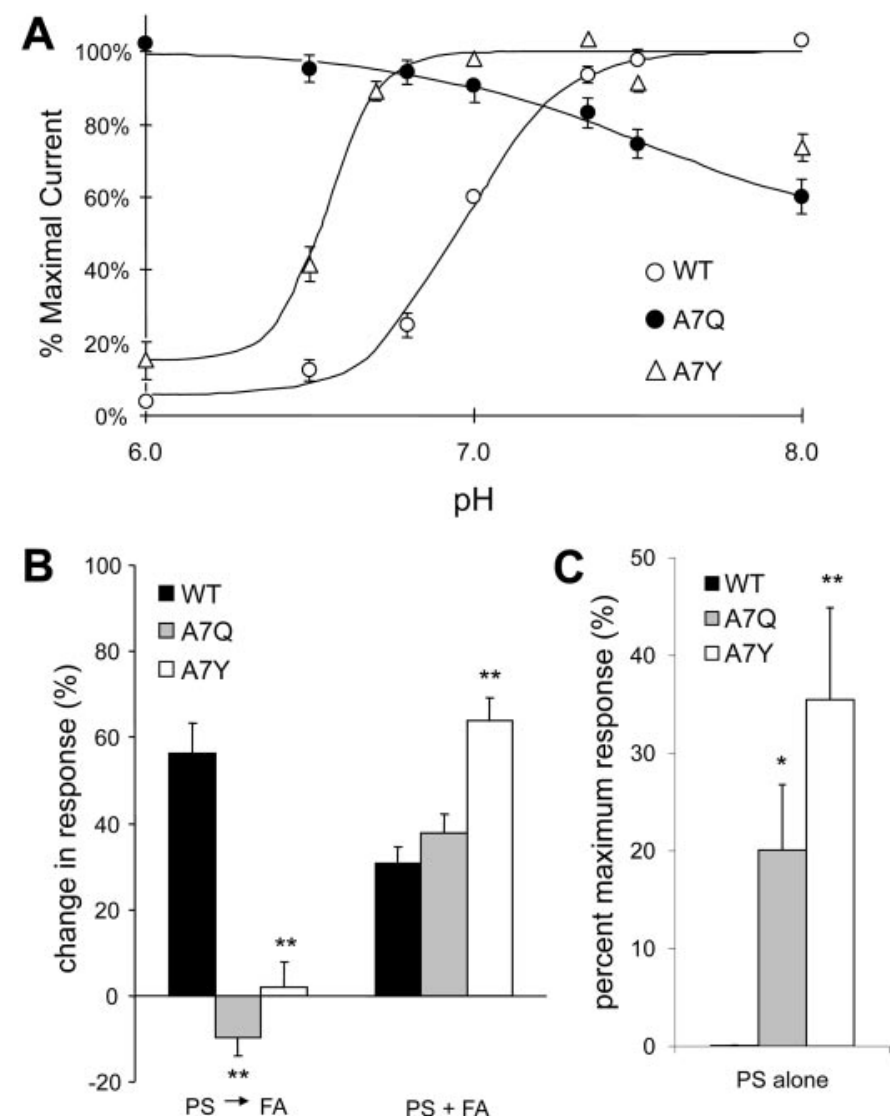

Fig. 8. Altered response of $A 7 Q$ and $A 7 Y$ to allosteric modulators. A proton inhibition curves in the presence of $100 \mu \mathrm{M}$ glycine and $100 \mu \mathrm{M}$ glutamate. WT receptors are highly sensitive to $\mathrm{pH}$ modulation, with a $\mathrm{p} K_{\mathrm{a}}$ value of 6.97. Decreased proton inhibition was observed in A7Y $\left(\mathrm{p} K_{\mathrm{a}}=6.59\right)$, whereas $\mathrm{A} 7 \mathrm{Q}$ was insensitive to proton block and inhibited by high $\mathrm{pH}$. Inhibition of A7Y was also seen at high $\mathrm{pH}$ values, which were not used in calculating the $\mathrm{p} K$ for that mutant. B, current potentiation by $100 \mu \mathrm{M}$ PS, applied either before or in the presence of agonist. PS preapplication yielded $\sim 50 \%$ potentiation of the WT full agonist response but had little effect on A7Q or A7Y (WT: $56 \pm 7 \%$; A7Q: $-10 \pm$ 5\%; and A7Y: $2 \pm 6 \%$ ). However, concurrent treatment with PS and full agonist restored potentiation in both mutants (WT: $31 \pm 4 \%$; A7Q: $38 \pm$ 4\%; and A7Y: $64 \pm 5 \%$ ). C, application of $100 \mu \mathrm{M}$ PS was found to partially activate both $\mathrm{A} 7 \mathrm{Q}$ and $\mathrm{A} 7 \mathrm{Y}$ even in the absence of agonist, whereas having no effect on WT (WT: $0.1 \pm 0.0 \%$; A7Q: $16 \pm 6 \%$; and A7Y: $30 \pm 10 \%$ ). $*, p<0.05$ (significant); $* *, p<0.01$ (highly significant). 